Mon. Not. R. Astron. Soc. 000, 000-000 (0000) Printed 29 October $2018 \quad$ (MN LATEX style file v2.2)

\title{
Two-dimensional kinematics of SLACS lenses - II. Combined lensing and dynamics analysis of early-type galaxies at $z=0.08-0.33$
}

\author{
Matteo Barnabè ${ }^{1 \star}$, Oliver Czoske ${ }^{1}$, Léon V. E. Koopmans ${ }^{1}$, Tommaso Treu ${ }^{2}$, \\ Adam S. Bolton ${ }^{3}$ and Raphaël Gavazzi ${ }^{4}$ \\ ${ }^{1}$ Kapteyn Astronomical Institute, University of Groningen, PO Box 800, 9700 AV Groningen, the Netherlands \\ ${ }^{2}$ Department of Physics, University of California, Santa Barbara, CA 93101, USA \\ ${ }^{3}$ Institute for Astronomy, University of Hawaii, 2680 Woodlawn Drive, Honolulu, HI 96822-1897, USA \\ ${ }^{4}$ Institut d'Astrophysique de Paris, CNRS, UMR 7095, Université Pierre et Marie Curie, 98bis Bd Arago, 75014 Paris, France
}

Accepted 2009 April 18. Received 2009 March 30; in original form 2009 January 12.

\begin{abstract}
We present the first detailed analysis of the mass and dynamical structure of a sample of six early-type lens galaxies, selected from the Sloan Lens ACS Survey, in the redshift range $0.08 \lesssim z \lesssim 0.33$. Both Hubble Space Telescope (HST)/ACS high-resolution imaging and VLT VIMOS integral field spectroscopy are available for these systems. The galaxies are modelled - under the assumptions of axial symmetry and two-integral stellar distribution function-by making use of the CAULDRON code, which self-consistently combines gravitational lensing and stellar dynamics, and is fully embedded within the framework of Bayesian statistics. The principal results of this study are: (i) all galaxies in the sample are well described by a simple axisymmetric power-law profile for the total density, with a logarithmic slope $\gamma^{\prime}$ very close to isothermal $\left(\left\langle\gamma^{\prime}\right\rangle=1.98 \pm 0.05\right.$ and an intrinsic spread close to 5 per cent $)$ showing no evidence of evolution over the probed range of redshift; (ii) the axial ratio of the total density distribution is rounder than 0.65 and in all cases, except for a fast rotator, does not deviate significantly from the flattening of the intrinsic stellar distribution; (iii) the dark matter fraction within the effective radius has a lower limit of about 15 to 30 per cent; (iv) the sample galaxies are only mildly anisotropic, with $|\delta| \leqslant 0.16$; (v) the physical distinction among slow and fast rotators, quantified by the $v / \sigma$ ratio and the intrinsic angular momentum, is already present at $z \gtrsim 0.1$. Altogether, early-type galaxies at $z=0.08-0.33$ are found to be markedly smooth and almost isothermal systems, structurally and dynamically very similar to their nearby counterparts. This work confirms the effectiveness of the combined lensing and dynamics analysis as a powerful technique for the study of early-type galaxies beyond the local Universe.
\end{abstract}

Key words: gravitational lensing — galaxies: elliptical and lenticular, cD — galaxies: kinematics and dynamics - galaxies: structure.

\section{INTRODUCTION}

The currently favoured cosmological scenario, the so-called $\Lambda \mathrm{CDM}$ (cold dark matter) paradigm, has been remarkably successful at explaining the large scale structure of the Universe. In the non-linear regime, below several Mpc, however, the situation is less certain, and a full understanding of the galaxy formation and evolution processes remains a work in progress.

Within the standard paradigm, massive early-type galaxies are thought to be the end-product of hierarchical merging of lower mass galaxies, and to be embedded in extended dark matter haloes

\footnotetext{
^ E-mail: M.Barnabe@ astro.rug.nl
}

(e.g. Toomre 1977; White \& Frenk 1991; Barnes 1992; Cole et al. 2000). Numerical studies of merging galaxies (e.g. Naab et al. 2006; Jesseit et al. 2007) have managed to reproduce a number of observational characteristics of massive ellipticals, and have made clear that stringent tests of galaxy formation models require a detailed and reliable description of the intrinsic physical properties of real early-type galaxies, such as their mass density distribution and orbital structure. Furthermore, knowledge of how these galaxy properties evolve through time would provide much needed information and even stronger constraints on the theoretical predictions.

In the last decades, local early-type galaxies have been the object of substantial observational and modelling efforts. These studies have employed a variety of tracers ranging from stel- 
Table 1. Basic data for the six SLACS lens galaxies analyzed in this work. The data are taken from Treu et al. (2006) and Koopmans et al. (2006).

\begin{tabular}{|c|c|c|c|c|c|c|c|c|c|c|c|}
\hline Galaxy name & $z_{1}$ & $z_{\mathrm{s}}$ & $\begin{array}{c}\sigma_{\text {SDSS }} \\
\left(\mathrm{km} \mathrm{s}^{-1}\right)\end{array}$ & $\begin{array}{l}R_{\text {eff, } \mathrm{V}} \\
(\mathrm{kpc})\end{array}$ & $\begin{array}{c}M_{V} \\
(\mathrm{mag})\end{array}$ & $\begin{array}{l}R_{\text {eff, B }} \\
(\mathrm{kpc})\end{array}$ & $\begin{array}{c}M_{B} \\
(\mathrm{mag})\end{array}$ & $q_{\star, 2 \mathrm{D}}$ & $\begin{array}{l}\vartheta_{\mathrm{PA}, \star} \\
(\mathrm{deg})\end{array}$ & $\begin{array}{l}R_{\text {Einst }} \\
(\mathrm{kpc})\end{array}$ & $\begin{array}{c}M_{\text {Einst }} \\
\left(10^{10} M_{\odot}\right)\end{array}$ \\
\hline SDSS J0037-0942 & 0.1955 & 0.6322 & $265 \pm 10$ & $8.48 \pm 0.11$ & -23.11 & $8.67 \pm 0.19$ & -22.25 & 0.76 & 9.5 & 4.77 & 27.3 \\
\hline SDSS J0216-0813 & 0.3317 & 0.5235 & $332 \pm 23$ & $16.95 \pm 0.92$ & -23.94 & $17.28 \pm 1.20$ & -23.06 & 0.85 & 79.2 & 5.49 & 48.2 \\
\hline SDSS J0912+0029 & 0.1642 & 0.3240 & $313 \pm 12$ & $14.66 \pm 0.23$ & -23.41 & $15.49 \pm 0.39$ & -22.55 & 0.67 & 13.2 & 4.55 & 39.6 \\
\hline SDSS J0959+0410 & 0.1260 & 0.5349 & $212 \pm 12$ & $4.50 \pm 0.05$ & -21.45 & $4.75 \pm 0.03$ & -20.58 & 0.68 & 57.4 & 2.25 & 7.7 \\
\hline SDSS J1627-0053 & 0.2076 & 0.5241 & $275 \pm 12$ & $6.68 \pm 0.06$ & -22.68 & $6.13 \pm 0.09$ & -21.71 & 0.85 & 5.6 & 4.11 & 22.2 \\
\hline SDSS J2321-0939 & 0.0819 & 0.5324 & $236 \pm 7$ & $7.93 \pm 0.07$ & -22.59 & $8.47 \pm 0.11$ & -21.72 & 0.77 & 126.5 & 2.43 & 11.7 \\
\hline
\end{tabular}

Notes: For each SLACS system we list: the redshifts $z_{1}$ and $z_{\mathrm{s}}$ of the lens galaxy and of the background source, respectively; the velocity dispersion $\sigma_{\mathrm{SDSS}}$ measured from the $3^{\prime \prime}$ diameter SDSS fibre; the effective radius $R_{\text {eff }}$ and absolute magnitude $M$ determined by fitting de Vaucouleurs profiles to the $V$ - and $B$-band ACS images; the isophotal axis ratio $q_{\star, 2 \mathrm{D}}$; the position angle $\vartheta_{\mathrm{PA}, \star}$ of the major axis; the Einstein radius $R_{\text {Einst }}$ and the total mass $M_{\text {Einst }}$ enclosed, in projection, inside $R_{\text {Einst }}$.

lar kinematics (e.g. Saglia, Bertin \& Stiavelli 1992, Bertin et al. 1994, Franx et al. 1994, Carollo et al. 1995, Rix et al. 1997, Loewenstein \& White 1999, Gerhard et al. 2001, Borriello et al. 2003, Thomas et al. 2007 and the SAURON collaboration: see e.g. de Zeeuw et al. 2002, Emsellem et al. 2004, Cappellari et al. 2006) and kinematics of discrete tracers such as globular clusters (e.g. Mould et al. 1990; Côté et al. 2003) or planetary nebulae (e.g. Arnaboldi et al. 1996, Romanowsky et al. 2003; also in combination with the kinematics of stars: de Lorenzi et al. 2008) to hot X-ray gas (e.g. Fabbiano 1989; Matsushita et al. 1998; Fukazawa et al. 2006; Humphrey et al. 2006), usually finding evidence for a dark matter halo component and for a total mass density profile close to isothermal (i.e. $\rho_{\text {tot }} \propto r^{-2}$ ) in the inner regions.

On the other hand, because of the severe observational limitations, thorough studies of distant early-type galaxies (at redshift $z \gtrsim 0.1)$ are still in their infancy. Traditional analyses based on dynamics alone are hindered by the lack of tracers at large radii and by the mass-anisotropy degeneracy, i.e. a change in the mass profile of the galaxy or in the anisotropy of the velocity dispersion tensor can both determine the same effect in the measured velocity dispersion map. Higher-order velocity moments, which potentially allow one to disentangle this degeneracy by providing additional constraints (Gerhard 1993; van der Marel \& Franx 1993), can only be measured with sufficient accuracy in the inner parts of nearby galaxies with the current instruments. Fortunately, valuable additional information on distant early-type galaxies can be provided by gravitational lensing (see e.g. Schneider, Ehlers \& Falco 1992), when the galaxy happens to act as a gravitational lens with respect to a luminous background source at higher redshift. Strong gravitational lensing allows one to determine the total mass within the Einstein radius $R_{\text {Einst }}$ in an accurate and almost model-independent way Kochanek 1991) although, due to the mass-sheet or massslope degeneracies (Falco, Gorenstein \& Shapiro 1985); Wucknitz 2002), it does not permit the univocal recovery of the mass density profile of the lens.

Gravitational lensing and stellar dynamics are particularly effective when they are applied in combination to the analysis of distant early-type galaxies. The complementarity of the two approaches is such that the mass-sheet and massanisotropy degeneracies can to a large extent be disentangled and the mass profile of the lens galaxy can be robustly determined (see e.g. Koopmans \& Treu 2002, 2003, Treu \& Koopmans 2002, 2003, 2004, Barnabè \& Koopmans 2007, Czoske et al. 2008, Czoske, Barnabè \& Koopmans 2008, van de Ven et al. 2008, Trott et al. 2008). Recently, the dedicated Sloan Lens ACS Sur- vey (SLACS; Bolton et al. 2006; Treu et al. 2006; Koopmans et al. 2006; Gavazzi et al. 2007; Bolton et al. 2008; Gavazzi et al. 2008; Bolton et al. 2008; Treu et al. 2008) has discovered a large and homogeneous sample of 70 strong gravitational lense 1 , for the most part early-type galaxies, at redshift between $z \simeq 0.05$ and 0.51. Koopmans et al. 2006) have applied a joint analysis to the SLACS galaxies, finding an average total mass profile very close to isothermal with no sign of evolution to redshifts approaching unity (when including also systems from the Lenses Structure and Dynamics Survey, e.g. Treu \& Koopmans 2004). A limitation of the method used in those works is that lensing and dynamics are treated as independent problems, and all the kinematic constraints come from a single aperture-averaged value of the stellar velocity dispersion, which could potentially lead to biased results. Barnabè \& Koopmans 2007, hereafter BK07) have expanded the technique for the combined lensing and dynamics analysis into a more general and self-consistent method, embedded within the framework of Bayesian statistics. Implemented as the CAULDRON algorithm, under the only assumptions of axial symmetry and twointegral stellar distribution function (DF) for the lens galaxy, this method makes full use of all the available data sets (i.e. the surface brightness distribution of the lensed source, and the surface brightness and kinematic maps of the lens galaxy) in order to recover the lens structure and properties in the most complete and reliable way allowed by the data. The ideal testing-ground for an in-depth analysis is represented by a sub-sample of 17 SLACS lenses which have been observed with the VIMOS integral-field unit (IFU) mounted on the VLT as part of a pilot and a large programme (ESO programmes 075.B-0226 and 177.B-0682, respectively; PI: Koopmans), obtaining detailed two-dimensional kinematic maps (first and second velocity moments) in addition to the HST imaging data. The first joint study conducted with the caulDRON code of one of these systems, SDSS J2321 at $z=0.082$, has been presented in Czoske et al. (2008, hereafter C08).

In this paper, we extend the study of $\mathrm{C} 08$ to a total of six SLACS lenses for which kinematic data sets are now available: SDSS J0037，SDSS J0216，SDSS J0912，SDSS J0959 and SDSS J1627, in addition to the already mentioned SDSS J2321. This sample was chosen to cover a range in redshift, mass and importance of rotation which is representative of the SLACS sample. Since the SLACS galaxies have been shown to be statistically

\footnotetext{
1 A further 19 systems are possible gravitational lenses, but the multiple imaging is not secure.
} 
Two-dimensional kinematics of SLACS lenses - II.

Table 2. Observing log. The HR_Blue grism was used in programme 075.B0226, the HR_Orange in programme 177.B-0682.

\begin{tabular}{crrlc}
\hline Galaxy & $N_{\text {exp }}$ & $T_{\text {exp }}(\mathrm{s})$ & Grism & $\lambda_{\text {rest }}[\AA]$ \\
\hline SDSS J0037 & 33 & 18315 & HR_Blue & {$[3860,5175]$} \\
SDSS J0216 & 14 & 28840 & HR_Orange & {$[3875,5350]$} \\
SDSS J0912 & 12 & 6660 & HR_Blue & {$[3860,5295]$} \\
SDSS J0959 & 5 & 10300 & HR_Orange & {$[4600,6300]$} \\
SDSS J1627 & 12 & 24720 & HR_Orange & {$[4200,5940]$} \\
SDSS J2321 & 15 & 8325 & HS_Blue & {$[5350,5450]$} \\
\hline
\end{tabular}

indistinguishable from control samples in terms of any of their known observables, such as size, luminosity, surface brightness (Bolton et al.2008), location on the Fundamental Plane (Treu et al. 2006) and environment (Treu et al. 2008), we expect that the results of the combined lensing and dynamics analysis described in this work can be generalized to the massive early-type population, nicely complementing the work done by, e.g., the SAURON collaboration on lower redshift and lower mass early-type galaxies. Basic information on the six systems under study are listed in Table 1 The VIMOS and HST observations of these systems, together with a description of the data reduction, will be detailed in a forthcoming paper (Czoske et al., in preparation).

This paper is organized as follows: in Section 2 we give a brief overview of the available data sets. In Section 3 we recall the basic features of the CAULDRON algorithm and the adopted mass model. The results of the combined lensing and dynamics analysis of the SLACS subsample are presented in Sections 4 and 5 with the latter focusing on the recovered dynamical structure of the lenses. In Section 6 we summarize our findings and draw conclusions. Throughout this paper we adopt a concordance $\Lambda \mathrm{CDM}$ model described by $\Omega_{\mathrm{M}}=0.3, \Omega_{\Lambda}=0.7$ and $H_{0}=100 h \mathrm{~km} \mathrm{~s}^{-1} \mathrm{Mpc}^{-1}$ with $h=0.7$, unless stated otherwise.

\section{OVERVIEW OF THE DATA SETS}

\subsection{Spectroscopy}

Integral-field spectroscopy for seventeen lens systems was obtained using the integral-field unit of VIMOS on the VLT, UT3. All observations, split in Observing Blocks (OB) of roughly one hour, including calibration, were done in service mode.

Of the six systems analyzed in this paper, three were observed in the course of a normal ESO programme, a pilot, 075.B0226 (PI: Koopmans). For this programme we used the HR-Blue grism with a resolution of $\sigma=0.8 \AA$ (1.9 $\AA$ full width at half maximum, FWHM), covering an observed wavelength range of 4000 to $6200 \AA$. Each OB was split into three dithered exposures of 555 seconds each. For the large programme, we switched to the HR-Orange grism with mean resolution $\sigma_{\lambda}=0.78 \AA$, covering the range 5050 to $7460 \AA$. Only one long exposure of 2060 seconds was obtained for each observing block; the number of observing blocks was sufficient to fill in on gaps in the data due to bad instrument fibres through pointing-offset between subsequent OBs.

The data were reduced using the VIPGI package (Scodeggio et al. 2005; Zanichelli et al. 2005). For more details on the procedure and tests of the quality of the reduced data we refer to Czoske et al. (2008) and Czoske et al. (in preparation).

The kinematic parameters $v$ and $\sigma$ were determined from the individual spectra using a direct pixel-fitting routine. Compared to Czoske et al. (2008), we have made a number of modifications in the algorithm. In particular, we now use almost the entire wavelength range that is available from the spectra; noisy parts at the blue and red ends of the spectra were cut off. Due to the varying redshifts of the lenses, the rest-frame wavelength ranges and hence the spectral features that were used in the kinematic analysis varied from lens to lens. The template used was a spectrum of the K2 giant HR 19, taken from the Indo-US survey (Valdes et al. 2004). The native resolution of the template spectrum is $1 \AA$ FWHM ( $\left.\sigma_{\lambda}=0.42 \AA\right)$. The template is first smoothed to the instrumental resolution of the VIMOS spectra, corrected to the rest frame of the lens. Due to the low signal-to-noise ratio of the spectra from individual spaxels, we assume the line-of-sight velocity distribution (LOSVD) to be described by a Gaussian. Tests show that including Gauss-Hermite terms $h_{3}$ and $h_{4}$ (van der Marel \& Franx 1993) does not improve the fit in terms of $\chi^{2}$ per degree of freedom and does not yield robust results for $h_{3}$ and $h_{4}$ and consequently for the velocity moments $\bar{v}$ and $\overline{v^{2}}$ (see also Cappellari \& Emsellem 2004). The larger wavelength range requires us to modify the linear correction function used in Czoske et al. (2008) by multiplicative and additive polynomial corrections (following Kelson et al. 2000). Extensive testing shows that choosing polynomial orders of five ensures good fits for the continuum shape without affecting the structure of the small scale absorption features.

A number of spectral features that are not well reproduced by the stellar template are masked. This includes in particular the $\mathrm{Mg} \mathrm{b}$ line which is enhanced in the lens galaxy, possibly the result of an $[\alpha / \mathrm{Fe}]$ enhancement, as compared to the Galactic star HR 19 (Barth et al. 2002) and the Balmer lines which may be partially filled in by emission.

\subsection{Imaging}

We use Hubble Space Telescope (HST) imaging data from ACS and NICMOS to obtain information on the surface brightness distributions of the lens galaxies and the gravitationally lensed background galaxies.

ACS images taken through the F814W filter form the basis of the lens modelling. For four of the systems, we have deep (full-orbit) imaging (program 10494, PI: Koopmans); for the remaining two (SDSS J2321, SDSS J0037) we use single-exposure images from our snapshot program (10174, PI: Koopmans). Nonparametric elliptical B-spline models of the lens galaxies were subtracted off the images in order to obtain a clean representation of the source structure without contamination from the lens galaxy (Bolton et al. 2008).

For the dynamical analysis the kinematic maps are weighted by the surface brightness of the lens galaxy. We use the reddest band possible since this gives the most reliable representation of the stellar light. For four of the six systems described here, NICMOS images taken through the F160W filter are available. For SDSS J2321 and SDSS J1627, we had instead to resort to the F814W ACS images. Since the lensed source is typically stronger in F814W than in F160W, we start from the B-spline model of the lens galaxy to which we add random Gaussian noise according to the variance map of the images. The images are convolved to the spatial resolution of the VIMOS data (the seeing limit of the ser- 

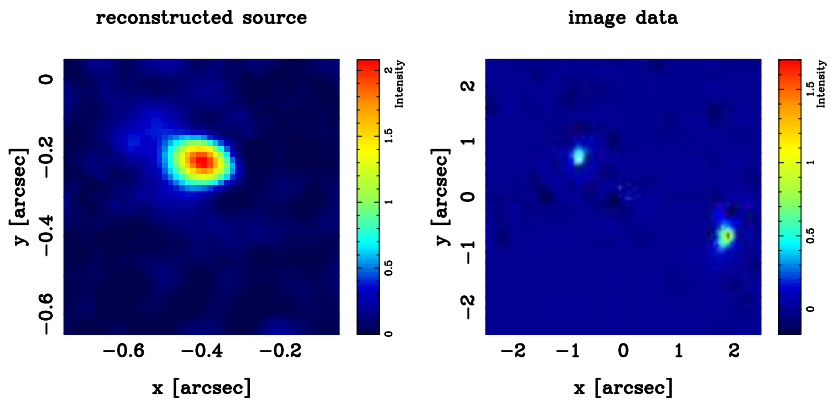

reconstructed image
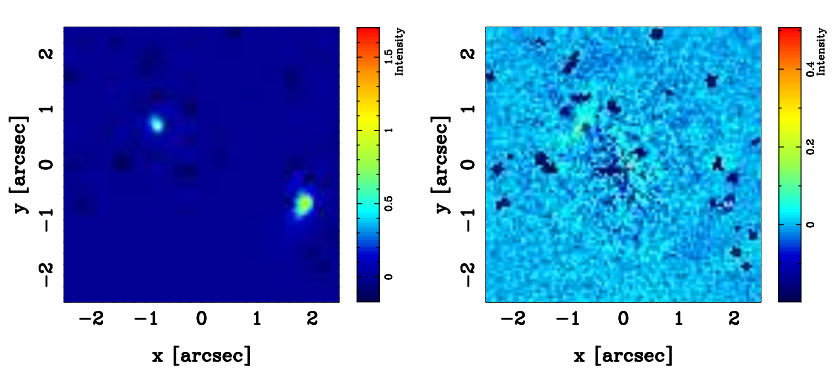

Figure 1. Best model lensed image reconstruction for the system SDSS J0037. From the top left-hand to bottom right-hand panel: reconstructed source model; HST/ACS data showing the lensed image after subtraction of the lens galaxy; lensed image reconstruction; residuals. In the panels, North is up and East is to the left.
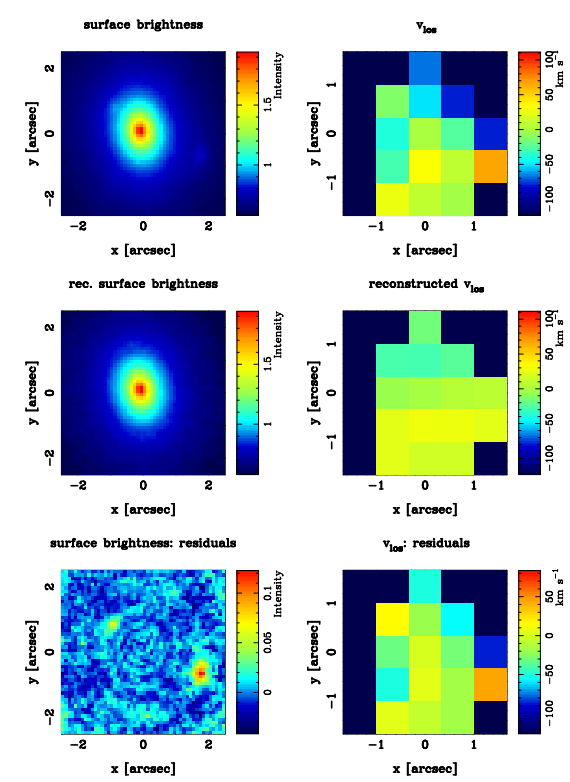

Figure 2. Best dynamical model for the galaxy SDSS J0037. First row: observed surface brightness distribution, projected line-of-sight velocity and line-of-sight velocity dispersion. Second row: corresponding reconstructed quantities for the best model. Third row: residuals. In the panels, North is up and East is to the left.

vice mode observations, $0.8 \mathrm{arcsec}$ ) and resampled to the grid of the kinematic data using swarn 2 (Bertin 2008).
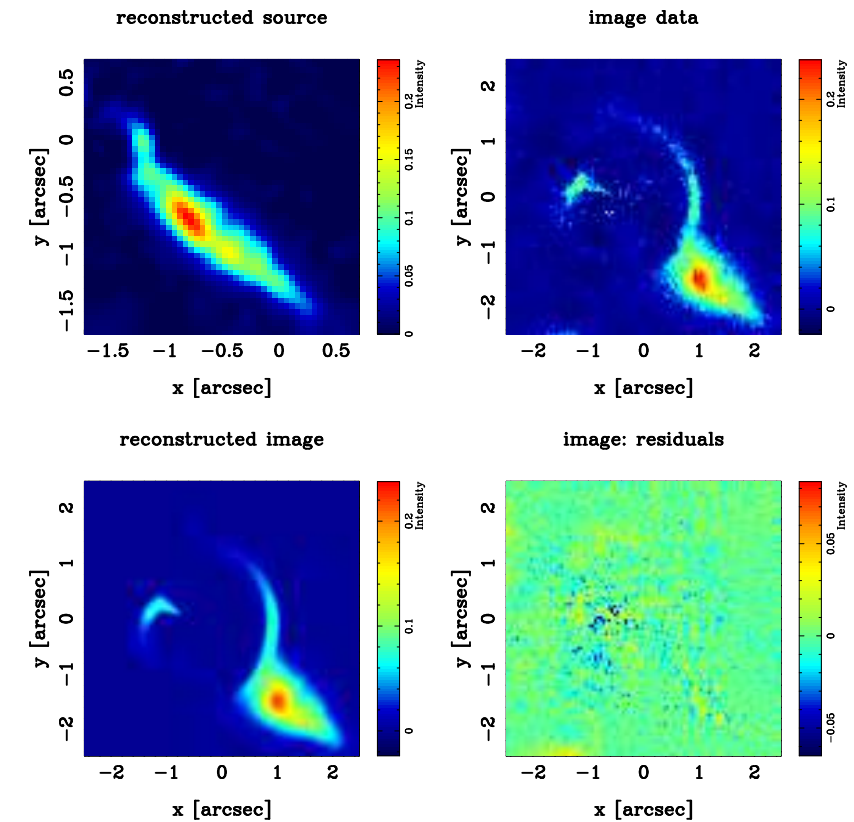

Figure 3. Best model lensed image reconstruction for the galaxy SDSS J0216. Panels meaning as in Fig. 1]

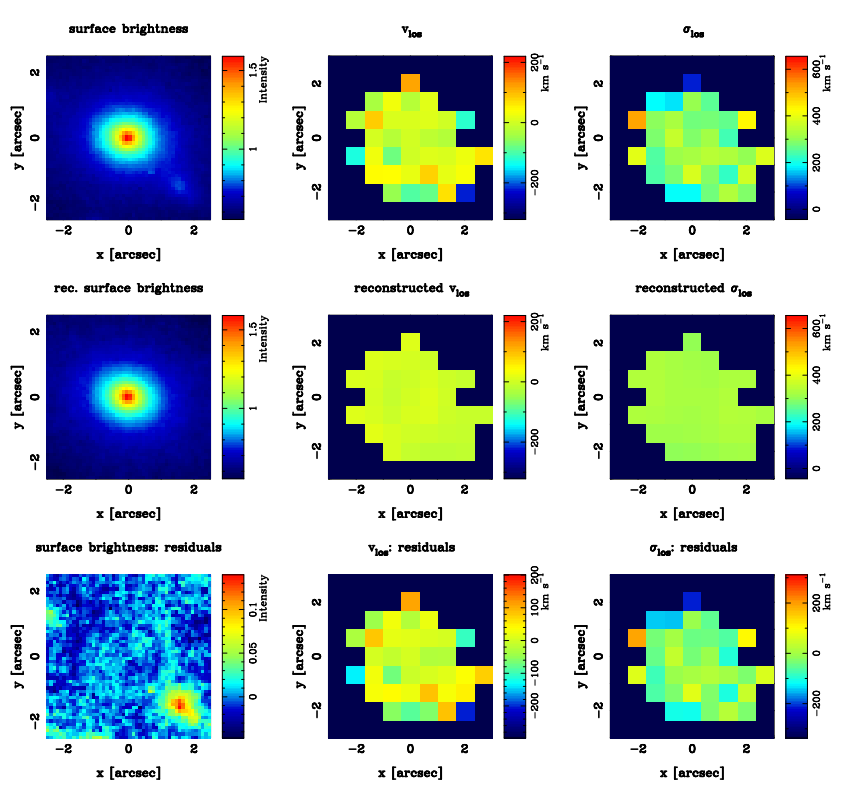

Figure 4. Best dynamical model for the galaxy SDSS J0216. Panels meaning as in Fig. 2

\section{COMBINING LENSING AND DYNAMICS}

In this Section we recall the main features of the CAULDRON algorithm and describe the adopted family of galaxy models. The reader is referred to BK07 for a detailed description of the method.

\subsection{Overview of the CAULDRON algorithm}

The central premise of a self-consistent joint analysis is to adopt a total gravitational potential $\Phi$ (or, equivalently, the total density profile $\rho$, from which $\Phi$ is calculated via the Poisson equation), parametrized by a set $\boldsymbol{\eta}$ of non-linear parameters, and use it simultaneously for both the gravitational lensing and the stellar 

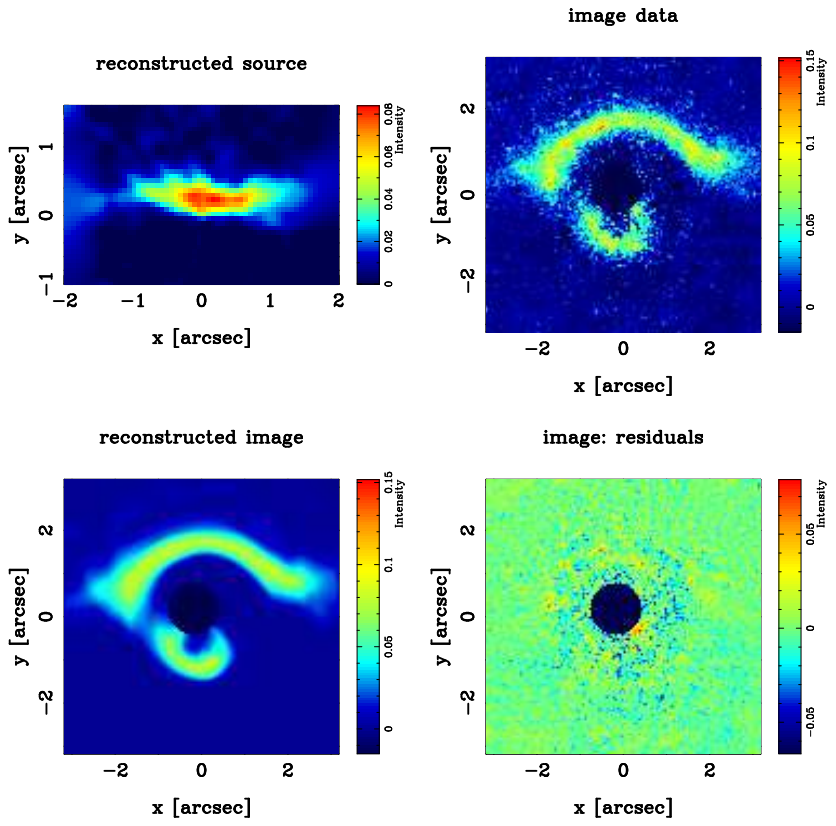

Figure 5. Best model lensed image reconstruction for the galaxy SDSS J0912. Panels meaning as in Fig. 1

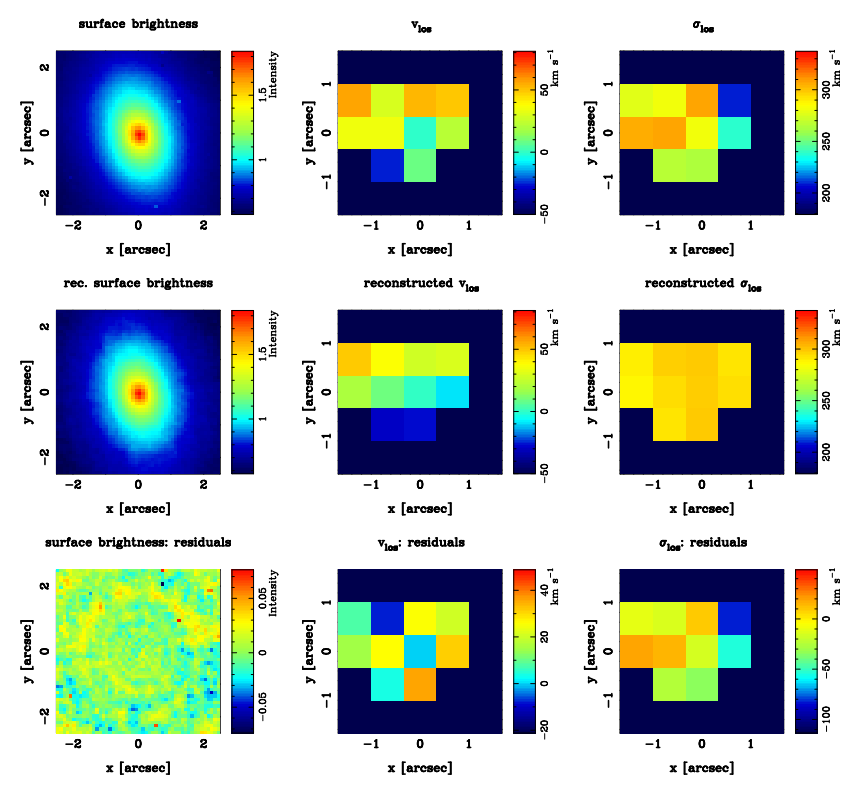

Figure 6. Best dynamical model for the galaxy SDSS J0912. Panels meaning as in Fig.2

dynamics modelling of the data. While different from a physical point of view, these two modelling problems can be formally expressed in an analogous way as a single set of coupled (regularized) linear equations. For any given choice of the non-linear parameters, the equations can be solved (in a direct, non-iterative manner) to obtain as the best solution for the chosen potential model: (i) the surface brightness distribution of the unlensed source, and (ii) the weights of the elementary stellar dynamics building blocks (e.g. orbits or two-integral components, TICs, Schwarzschild 1979; Verolme \& de Zeeuw 2002). This linear optimization scheme is consistently embedded within the framework of Bayesian statistics. As a consequence, it is possible to objectively assess the probability of each model by means of the evidence merit function
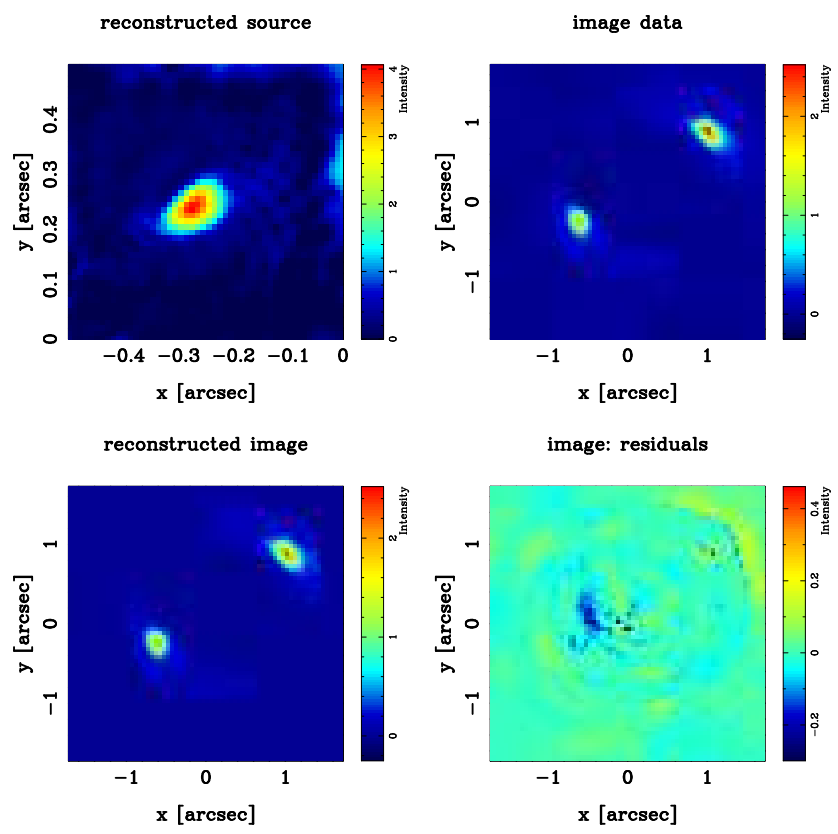

Figure 7. Best model lensed image reconstruction for the galaxy SDSS J0959. Panels meaning as in Fig. 1
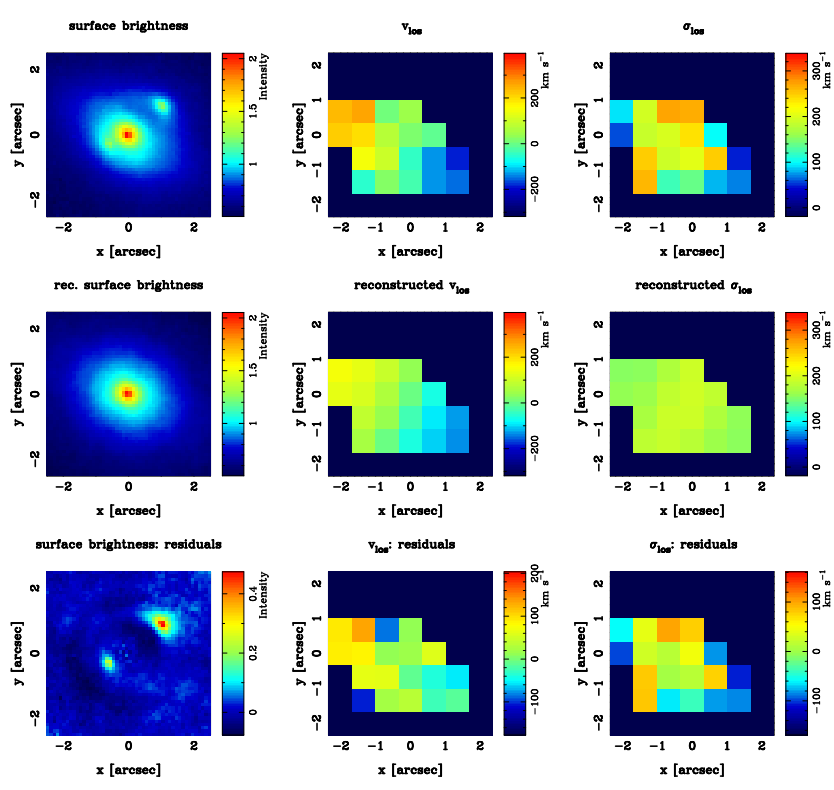

Figure 8. Best dynamical model for the galaxy SDSS J0959. Panels meaning as in Fig. 2

(also called the marginalized likelihood) and, therefore, to compare and rank different models (see MacKay 1992, 1999, 2003). In this way, by maximizing the evidence, one recovers the set of non-linear parameters $\boldsymbol{\eta}$ corresponding to the "best" potential (or density) model, i.e. that model which maximizes the joint posterior probability density function (PDF), hence called maximum $a$ posteriori (MAP) model. In the context of Bayesian statistics, the MAP model is the most plausible model in an Occam's razor sense, given the data and the adopted form of the regularization (the optimal level of the regularization is also set by the evidence). However, in a Bayesian context, the MAP parameters individually do not necessarily have to be probable: they have a high joint probability density, but might only occupy a small volume in parameter space. 

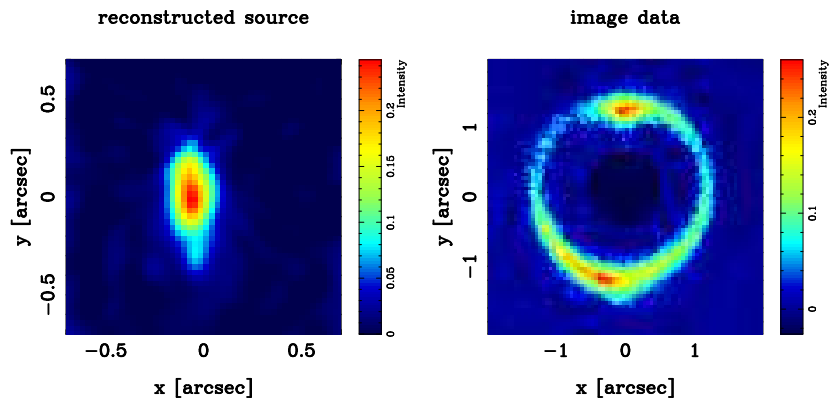

reconstructed image
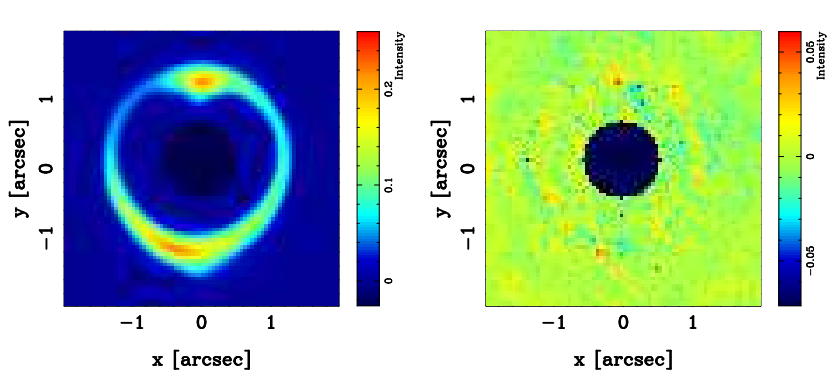

Figure 9. Best model lensed image reconstruction for the galaxy SDSS J1627. Panels meaning as in Fig. 1
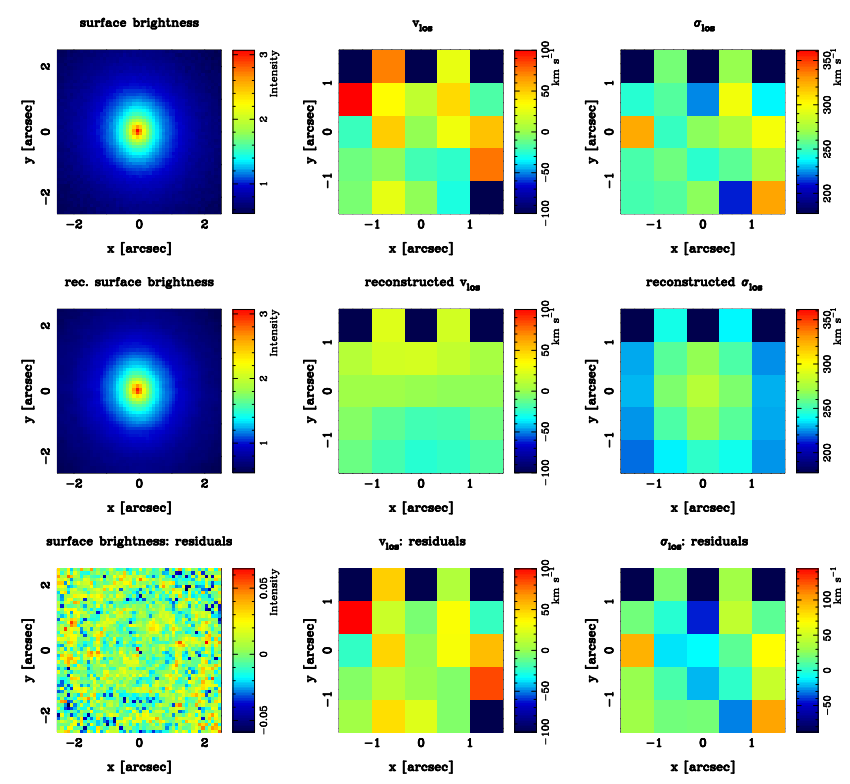

Figure 10. Best dynamical model for the galaxy SDSS J1627. Panels meaning as in Fig.2

This situation can arise if the MAP solution does not lie in the bulk of the posterior probability distribution (roughly parameter-space volume times likelihood density). This can be particularly severe if the PDF is non-symmetric in a high-dimensional space where volume can dramatically increase with distance from the MAP solution. In that case, the MAP solution for each parameter could easily lie fully outside the PDFs of each individual parameter, when marginalized over all other parameters. We will see this happening in some cases, and, although somewhat counter-intuitive, it is fully consistent with Bayesian statistics and a peculiar aspect of statistics in high-dimensional spaces.

As discussed in BK07, the method is extremely general and can in principle be applied to any potential shape. However, its current practical implementation, the CAULDRON algorithm, is more restricted in order to make it computationally efficient and applies specifically to axially symmetric potentials, $\Phi(R, z)$, and twointegral DFs $f=f\left(E, L_{z}\right)$, where $E$ and $L_{z}$ are the two classical integrals of motion, i.e., respectively, energy and angular momentum along the rotation axis. Under these assumptions, the dynamical model can be constructed by making use of the fast Monte Carlo numerical implementation by BK07 of the twointegral Schwarzschild method described by Cretton et al. (1999) and Verolme \& de Zeeuw (2002), whose building blocks are not stellar orbits (as in the classical Schwarzschild method) but TICs 3 The weights map of the optimal TIC superposition that best reproduces the observables, in a Bayesian sense, is yielded as an outcome of the joint analysis.

The code is remarkably robust and its applicability is not drastically limited by these assumptions. Barnabè et al. (2008) have tested CAULDRON on a galaxy model (comprising a stellar component and a dark matter halo) resulting from a numerical N-body simulation of a merger process, i.e. a complex non-symmetrical system which departs significantly from the algorithm's assumptions. Despite this, it is found that several important global properties of the system, including the total density slope and the dark matter fraction, are reliably recovered.

\subsection{The galaxy model}

Koopmans et al. (2006) have shown that a power-law model, despite its simplicity, seems to provide a satisfactory description for the total mass density profile of the inner regions of SLACS lens galaxies, to the level allowed by the data. This has been further confirmed by the C08 study of SDSS J2321, the first case where a fully self-consistent analysis was performed. Therefore, in the present work, we still adopt a power-law model. If this description is oversimplified for the galaxy under analysis, this will usually have a clearly disruptive effect on the quality of the reconstruction, such as very large residuals (compared to the noise level) for the best model lensed image, and a patchy or strongly pixelized reconstructed source (Barnabè et al. 2008).

The total mass density distribution of the galaxy is taken to be a power-law stratified on axisymmetric homoeoids:

$\rho(m)=\frac{\rho_{0}}{m^{\gamma^{\prime}}}, \quad 0<\gamma^{\prime}<3$,

where $\rho_{0}$ is a density scale, $\gamma^{\prime}$ will be referred to as the (logarithmic) slope of the density profile, and

$m^{2}=\frac{R^{2}}{a_{0}^{2}}+\frac{z^{2}}{c_{0}^{2}}=\frac{R^{2}}{a_{0}^{2}}+\frac{z^{2}}{a_{0}^{2} q^{2}}$,

where $c_{0}$ and $a_{0}$ are length-scales and $q \equiv c_{0} / a_{0}$.

The (inner) gravitational potential associated with a homoeoidal density distribution $\rho(m)$ is given by Chandrasekhar (1969) formula. In our case, for $\gamma^{\prime} \neq 2$, one has

$\Phi(R, z)=-\frac{\Phi_{0}}{\gamma^{\prime}-2} \int_{0}^{\infty} \frac{\tilde{m}^{2-\gamma^{\prime}}}{(1+\tau) \sqrt{q^{2}+\tau}} \mathrm{d} \tau$,

\footnotetext{
3 A TIC can be visualized as an elementary toroidal system, completely specified by a particular choice of energy $E$ and axial component of the angular momentum $L_{z}$. TICs have simple $1 / R$ radial density distributions and analytic unprojected velocity moments, and by superposing them one can build $f\left(E, L_{z}\right)$ models for arbitrary spheroidal potentials (cf. Cretton et al. 1999): all these characteristics contribute to make TICs particularly valuable and inexpensive building blocks when compared to orbits.
} 
Table 3. Recovered parameters and quantities for the best power-law models of the six analyzed SLACS lens galaxies.

\begin{tabular}{lccccccccc}
\hline Galaxy name & $\begin{array}{c}i \\
(\mathrm{deg})\end{array}$ & $\alpha_{0}$ & $\gamma^{\prime}$ & $q$ & $\begin{array}{c}\vartheta_{\mathrm{PA}} \\
(\mathrm{deg})\end{array}$ & $f_{\mathrm{dm}}\left(R_{\mathrm{e}} / 2\right)$ & $f_{\mathrm{dm}}\left(R_{\mathrm{e}}\right)$ & $\begin{array}{c}M_{\mathrm{e}} \\
\left(10^{11} M_{\odot}\right)\end{array}$ & $(M / L)_{\odot, B}$ \\
\hline SDSS J0037-0942 & $65^{\circ} .6$ & 0.434 & 1.968 & 0.693 & 8.8 & 0.10 & 0.23 & 3.35 & 5.40 \\
SDSS J0216-0813 & $70^{\circ} 0$ & 0.344 & 1.973 & 0.816 & $76^{\circ} 6$ & 0.19 & 0.17 & 12.20 & 9.35 \\
SDSS J0912+0029 & $87^{\circ} .8$ & 0.412 & 1.877 & 0.672 & 13.3 & 0.16 & 0.30 & 7.40 & 9.08 \\
SDSS J0959+0410 & $80^{\circ} .4$ & 0.323 & 1.873 & 0.930 & 64.2 & 0.25 & 0.30 & 0.95 & 7.17 \\
SDSS J1627-0053 & $56^{\circ} .4$ & 0.369 & 2.122 & 0.851 & 7.3 & 0.11 & 0.21 & 2.23 & 5.93 \\
SDSS J2321-0939 & $67^{\circ} .8$ & 0.468 & 2.061 & 0.739 & $135^{\circ} .5$ & 0.13 & 0.29 & 1.98 & 5.22 \\
\hline
\end{tabular}

Notes: We list: the four non-linear parameters, i.e. the inclination $i$, the lens strength $\alpha_{0}$, the logarithmic slope $\gamma^{\prime}$ and the axial ratio $q$; the position angle $\vartheta_{\mathrm{PA}}$; the dark matter fraction $f_{\mathrm{dm}}$ within a spherical shell of radius 0.5 and $1 R_{\mathrm{e}}$ (respectively), obtained under the maximum bulge hypothesis; the upper limit for the luminous mass $M_{\mathrm{e}}$ contained inside $R_{\mathrm{e}}$; the upper limit for the mass-to-light ratio in the $B$-band.

while for $\gamma^{\prime}=2$

$\Phi(R, z)=\Phi_{0} \int_{0}^{\infty} \frac{\log \tilde{m}}{(1+\tau) \sqrt{q^{2}+\tau}} \mathrm{d} \tau$,

where $\Phi_{0}=2 \pi G q a_{0}^{2} \rho_{0}$ and

$\tilde{m}^{2}=\frac{R^{2}}{a_{0}^{2}(1+\tau)}+\frac{z^{2}}{a_{0}^{2}\left(q^{2}+\tau\right)}$.

There are three non-linear parameters in the potential to be determined via the evidence maximization: $\Phi_{0}$ (or equivalently, through equation [B4] of BK07, the adimensional lens strength $\alpha_{0}$ ), the logarithmic slope $\gamma^{\prime}$ and the axial ratio $q$. A core radius $R_{\mathrm{S}}$ can be straightforwardly included in the density distribution, if necessary. Beyond these parameters, there are four additional parameters which determine the geometry of the observed system: the position angle $\vartheta_{\mathrm{PA}}$, the inclination $i$ and the coordinates of the lens galaxy centre with respect to the sky grid. Usually, the position angle and the lens centre (which are very well constrained by the lensed image brightness distribution) can be accurately determined by means of fast preliminary explorations and kept fixed afterwards in order to reduce the number of free parameters during the more computationally expensive optimization and error analysis runs. Finally, a proper modelling of the lensed image can occasionally require the introduction of two additional parameters, namely the shear strength and the shear angle, in order to account for external shear.

We employ a curvature regularization (defined as in Suyu et al. 2006 and Appendix A of BK07) for both the gravitational lensing and the stellar dynamics reconstructions. The level of the regularization is controlled by three so-called "hyperparameters" (one for lensing and two for dynamics, see discussion in BK07), whose optimal values are also set via maximization of the Bayesian evidence. The starting values of the hyperparameters are chosen to be quite large, since the convergence to the maximum is found to be faster when starting from an overregularized system.

\section{ANALYSIS AND RESULTS}

\subsection{Best model reconstruction}

We have applied the combined CAULDRON code to the analysis of the six SLACS lens galaxies in our current sample with available kinematic maps. The recovered non-linear parameters for each galaxy best model are presented in Table 3 The listed parameters are the inclination $i$ (expressed in degrees), the lens strength $\alpha_{0}$, the logarithmic density slope $\gamma^{\prime}$ and the axial ratio $q$ of the total density distribution. The Bayesian statistical errors on the parameters (i.e. the posterior probability distributions) are presented in Section 4.3

Our analysis shows that, given the current data, there is no need to include external shear or core radius in the modelling of any of the six galaxies: there is no significant improvement in the evidence when these parameters are allowed to vary, and their final values are found to be very close to zero. As mentioned in Section 3 for each system the lens centre and position angle are evaluated in a preliminary run and then kept constant. The best model position angles, relative to the total mass distribution, are found to depart less than $10^{\circ}$ (and, with the exception of SDSS J0959 and SDSS J2321, less than $3^{\circ}$ ) from the observed values obtained from the light distribution. This suggests that there is at most a small misalignment between the dark and luminous components. A similar conclusion was also drawn in Koopmans et al. (2006) and used to set an upper limit on the level of external shear.

The reconstructed observables corresponding to the best model for each galaxy (with the exception of SDSS J2321, for which we refer to the plots presented in C08) are shown and compared to the data in Figs 1 to 10 The reconstruction of both lensing and kinematic quantities appears in general to be very accurate. The residuals in the reconstructed lensed image are typically consistent with the noise level, and there is no sign of substantial discrepancies: this indicates that the underlying total density distribution is not significantly more complex (e.g. strongly triaxial) than the adopted axisymmetric power-law model, as discussed in Barnabè et al. (2008). The surface brightness distribution is also well reconstructed. The low-level ripples which are sometimes visible in the residuals map are due to the discrete nature of the TICs, and can be easily remedied by increasing the number of the employed TIC components, at the cost of a considerable slow-down of the optimization process, and without changing significantly the results. In the case of SDSS J0037, SDSS J0216 and SDSS J0959, the residuals clearly reveal the presence of the lensed image, which is not prominent in the surface brightness data map. The situation is more complicated for the kinematic maps, where the noise level is higher and the data sets composed by a smaller number of pixels (with as few as ten pixels for SDSS J0912). The models are fairly successful in reproducing the observables, in particular the velocity maps, with the exception of the velocity dispersion maps of SDSS J1627 (the reconstructed $\sigma_{\text {los }}$ profile declines too rapidly) and possibly SDSS J0959 (there appears to be a stronger gradient in the data than in the model). Such difficulties in reproducing the velocity dispersion maps of these two systems might well reflect the shortcomings of the two-integral DF assumption (which im- 

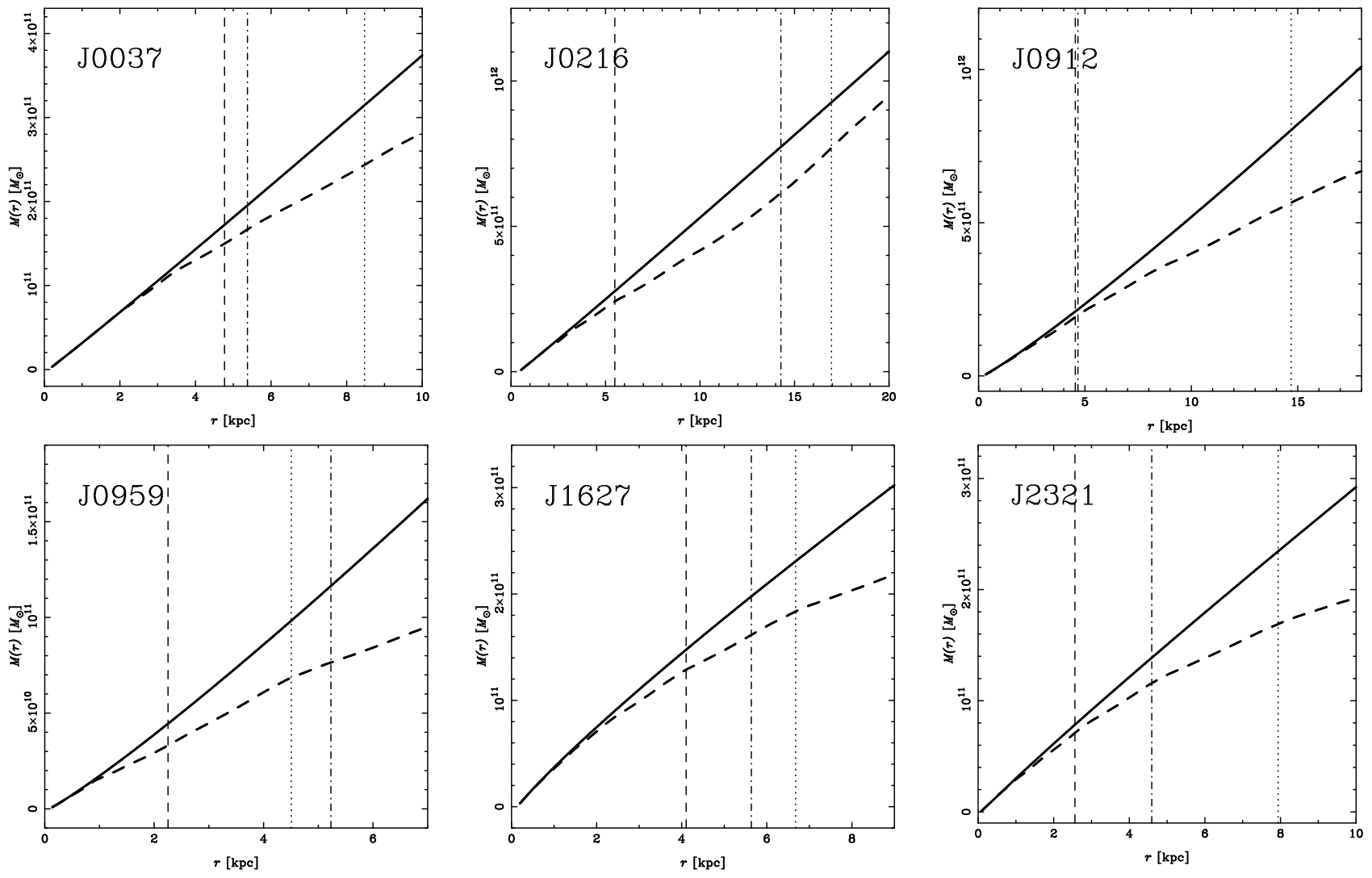

Figure 11. Spherically averaged mass distributions for the sample galaxies. Thick solid line: total mass profile obtained from the best reconstructed model; thick dashed line: luminous mass profile (calculated under the maximum bulge hypothesis); vertical dotted line: effective radius; vertical dashed line: Einstein radius; vertical dash-dotted line: outermost boundary of the kinematic data.

plies isotropic velocity dispersion tensor in the meridional plane, i.e. $\sigma_{R}=\sigma_{z}$ ), whereas a more sophisticated three-integral dynamical modelling could be required. We discuss this issue further in Section 6

\subsection{Mass distribution and dark matter fraction}

For each galaxy, we have calculated from the best model the spherically averaged total mass profile, shown in Fig. 11. In order to assess the dark matter content of the analyzed systems, one also requires the corresponding profile for the luminous component, which can be obtained from the best-model reconstructed stellar DF. However, within the framework of the method, stars are just tracers of the total potential, and therefore an additional assumption is needed in order to constrain normalization for the luminous mass distribution (which is arbitrary unless the stellar mass-to-light ratio can be determined independently). Following C08, in this work we adopt the so-called maximum bulge approach, which consists of maximizing the contribution of the luminous component. In other words, the stellar density profile is maximally rescaled without exceeding the total density profile 4 , positing a position-independent stellar mass-to-light ratio. In real galaxies the stellar mass-to-light

4 This approach is effectively the early-type galaxies equivalent of the classical maximum disk hypothesis van Albada \& Sancisi 1986) frequently used in modelling the rotation curves of late-type galaxies. ratio might not be uniform, although, based on observed colour gradients, the effect is not expected to be strong (e.g. Kronawitter et al. 2000). This procedure provides a uniform way to determine a lower limit for the dark matter fraction in the sample galaxies. Moreover, as shown in Barnabè et al. (2008), the maximum bulge approach is quite robust and allows a reliable determination of the dark matter fraction (within approximately 10 per cent of the total mass) even when the model assumptions are violated. The spherically averaged stellar mass profiles obtained under the maximum bulge hypothesis are also presented in Fig. 11 where we also indicate the three-dimensional radii corresponding to the effective radius $R_{\mathrm{e}}$, the Einstein radius $R_{\text {Einst }}$ and the outermost boundary of the kinematic data $R_{\text {kin }}$. The boundary of the surface brightness map is at least comparable to $R_{\text {kin }}$, and often larget ${ }^{5}$. Although the spatial coverage of the lensing and kinematic data, in most cases, does not extend up to $R_{\mathrm{e}}$ (the only exception being SDSS J0959, for which $R_{\text {kin }} \sim 1.2 R_{\mathrm{e}}$ ), it should be noted that the more distant regions of the galaxy which are situated along the line of sight-and therefore observed in projection-also contribute fairly significantly in constraining the mass model, as extensively discussed in C08.

We find that the total mass profile closely follows the light in the very inner regions, which are presumably dominated by the stellar component, while dark matter typically starts playing a role

\footnotetext{
5 The surface brightness maps considered for the combined analysis and presented in Figs 20 6 8 and 10 are actually cut-outs of $H S T$ images extending over 10 arcsec.
} 
in the vicinity of the (three-dimensional) radius $r=R_{\mathrm{e}} / 2$, where its contribution in total mass is of order 10 to 25 per cent, and becomes progressively more important when moving outwards (the system SDSS J0216, however, constitutes an exception, with its dark matter fraction remaining roughly constant over the probed region for $r \gtrsim 10 \mathrm{kpc}$ ). Within a sphere of radius $r=R_{\mathrm{e}}$, approximately 15 to 30 per cent of the mass is dark. This result is in general agreement with the conclusions of previous dynamical studies of early-type galaxies in the local Universe, in particular the analysis of 21 nearly round and slowly-rotating ellipticals by Gerhard et al. (2001), the modelling of 25 SAURON systems (under the assumption that mass follows light, Cappellari et al. 2006), and the study by Thomas et al. (2007) of 17 early-type galaxies in the Coma cluster.

From the value $M_{\mathrm{e}}$ of the luminous mass inside the effective radius, obtained under the maximum bulge hypothesis, we also calculate for each system the corresponding upper limit for the stellar mass-to-light ratio (see Table 3), finding $5 \lesssim(M / L)_{\odot . B} \lesssim 9$. This is in agreement with stellar population studies, e.g. Truiillo et al. (2004).

\subsection{Error analysis}

In this Section we present, for each galaxy in the sample, the corresponding model uncertainties, i.e. the errors on the recovered non-linear parameters $i, \alpha_{0}, \gamma^{\prime}$ and $q$. The uncertainties are calculated within the framework of Bayesian statistics by making use of the recently developed nested sampling technique (Skilling 2004, Sivia \& Skilling 2006; see also Vegetti \& Koopmans 2008 for the first astrophysical application in the context of gravitational lensing). Nested sampling is a Monte Carlo method aimed at calculating the Bayesian evidence, i.e. the fundamental quantity for model comparison, in a computationally efficient way. The marginalized posterior probability distribution functions (PDFs) of the model parameters, which are used to estimate the uncertainties, are obtained as very valuable by-products of the method.

Within the context of Bayesian statistics, a priori assumptions or knowledge on each parameter $\eta_{\mathrm{i}}$ are made explicit and formalized by defining the prior function $p\left(\eta_{\mathrm{i}}\right)$. We assign a uniform prior within the interval $2 \delta \eta_{\mathrm{i}}$, symmetrical around the recovered best model value $\eta_{\mathrm{b}, \mathrm{i}}$ and wide enough to include the bulk of the likelihood (very conservative estimates of $\delta \eta_{\mathrm{i}}$ are obtained by means of fast preliminary runs), that is:

$p\left(\eta_{\mathrm{i}}\right)=\left\{\begin{array}{lll}\text { constant } & \text { for } & \left|\eta_{\mathrm{b}, \mathrm{i}}-\eta_{\mathrm{i}}\right| \leqslant \delta \eta_{\mathrm{i}} \\ 0 & \text { for } & \left|\eta_{\mathrm{b}, \mathrm{i}}-\eta_{\mathrm{i}}\right|>\delta \eta_{\mathrm{i}} .\end{array}\right.$

This choice of an uniform prior is aimed at formalizing the absence of any a priori information within the interval $2 \delta \eta_{\mathrm{i}}$ (see e.g. Cousins 1995). We find, however, that the errors on the parameters are very small in comparison with $\delta \eta_{\mathrm{i}}$, so that the prior, largely independently of the adopted functional form, is nearly constant over the likelihood. Therefore, the specific choice for $p\left(\eta_{\mathrm{i}}\right)$ is not critical in our case.

For each analyzed galaxy, the histograms in Fig. 12 show the marginalized posterior PDF of the power-law model non-linear parameters. Because of the marginalization involved in their evaluation, these distributions constitute the most conservative estimate of statistical errors on the parameters, given the data and all the assumptions (i.e. positing that the adopted model is the "true" description underlying the data; cf. MacKay 1992). These errors are relatively small, as a consequence of the numerous constraints provided by the data: the typical data set for each of the sample galaxies consists of $\sim 10^{4}$ data points or more, most of them (in the lensed image and surface brightness maps) characterized by fairly good signal to noise level. Furthermore, the maximum $\eta_{\mathrm{i}, \mathrm{mp}}$ of the posterior PDF (and, more generally, the bulk of the posterior probability) for the $i$-th parameter is often found to be somewhat skewed with respect to the corresponding best model value $\eta_{\mathrm{i}, \mathrm{b}}$. This is a well-known projection effect arising from the marginalization over a single parameter of a complicated high-dimensional multivariate function such as the total posterior PDF, as discussed above $(\$ 3.1$.

The analysis conducted in this Section does not take into account systematic uncertainties, which are frequently larger than the statistical errors, and more difficult to quantify. They can arise from a variety of sources, including incorrect modelling assumptions and problems associated with the generation of the data sets (see e.g. Marshall et al. 2007 for an in-depth treatment of the systematic uncertainties connected with the lens galaxy subtraction process and the incomplete knowledge of the PSF). The study of Barnabè et al. (2008) provides a more quantitative feel for the systematic errors introduced by the adoption of an oversimplified galaxy model, showing that even in a quite extreme case (where the reconstruction of lensing observables is clearly unsatisfactory) the systematic error on $\gamma^{\prime}$ remains within about 10 per cent. Other parameters such as inclination and oblateness, however, are less robust and actually become ill-defined if the assumption of axial symmetry does not hold. We note, however, that in none of the six systems under study are the model residuals as severe as in the simulations in Barnabè et al. (2008). Therefore, we expect systematic uncertainties to remain within a few per cent level.

\subsection{The density profile of the ensemble}

From the combined analysis, we have found that all the galaxies in the ensemble have a total density profile very close to isothermal, with an average logarithmic slope $\left\langle\gamma^{\prime}\right\rangle=1.98 \pm 0.05$, in agreement with the results of Koopmans et al. (2006). There is also no evidence of evolution of the density slope with redshift (see Fig. 13.

No correlation is found between the logarithmic slope $\gamma^{\prime}$ and the axial ratio $q$, the effective radius, the normalized Einstein radius (i.e., the ratio $R_{\text {Einst }} / R_{\mathrm{e}}$ ) and the aperture averaged velocity dispersion $\sigma_{\text {SDSS }}$.

We now want to calculate, on the basis of the analyzed systems, the intrinsic spread around the average slope. If we assume that the slope $\gamma_{\mathrm{i}}^{\prime}$ of each galaxy is extracted from a parent Gaussian distribution of centre $\gamma_{\mathrm{c}}^{\prime}$ and variance $\sigma_{\gamma^{\prime}}$, then the joint posterior probability for the sampling is given by

$\mathcal{P}\left(\gamma_{\mathrm{c}}^{\prime}, \sigma_{\gamma^{\prime}} \mid\left\{\gamma_{\mathrm{i}}^{\prime}\right\}\right) \propto p\left(\gamma_{\mathrm{c}}^{\prime}, \sigma_{\gamma^{\prime}}\right) \prod_{\mathrm{i}} \frac{\exp \left[-\frac{\left(\gamma_{\mathrm{i}}^{\prime}-\gamma_{\mathrm{c}}^{\prime}\right)^{2}}{2\left(\sigma_{\gamma^{\prime}}^{2}+\delta \gamma_{\mathrm{i}}^{\prime 2}\right)}\right]}{\sqrt{2 \pi\left(\sigma_{\gamma^{\prime}}^{2}+\delta \gamma_{\mathrm{i}}^{\prime 2}\right)}}$,

where $p\left(\gamma_{\mathrm{c}}^{\prime}, \sigma_{\gamma^{\prime}}\right)$ is the prior on $\gamma_{\mathrm{c}}^{\prime}$ and $\sigma_{\gamma^{\prime}}$ (for which we adopt a uniform distribution) and $\delta \gamma_{i}^{\prime}$ are the $1 \sigma$ errors on $\gamma_{i}^{\prime}$, calculated by considering the region around the peak which contains 68 per cent of the posterior probability. From Eq. (7), the maximum likelihood solution for $\gamma_{\mathrm{c}}^{\prime}$ is simply the average slope $\left\langle\gamma^{\prime}\right\rangle$, while for $\sigma_{\gamma^{\prime}}$ is obtained from the equation

$\sum_{\mathrm{i}}\left[\frac{\left(\gamma_{\mathrm{i}}^{\prime}-\gamma_{\mathrm{c}}^{\prime}\right)^{2}-\sigma_{\gamma^{\prime}}^{2}-\delta \gamma_{\mathrm{i}}^{\prime 2}}{\left(\sigma_{\gamma^{\prime}}^{2}+\delta \gamma_{\mathrm{i}}^{\prime 2}\right)^{2}}\right]=0$ 

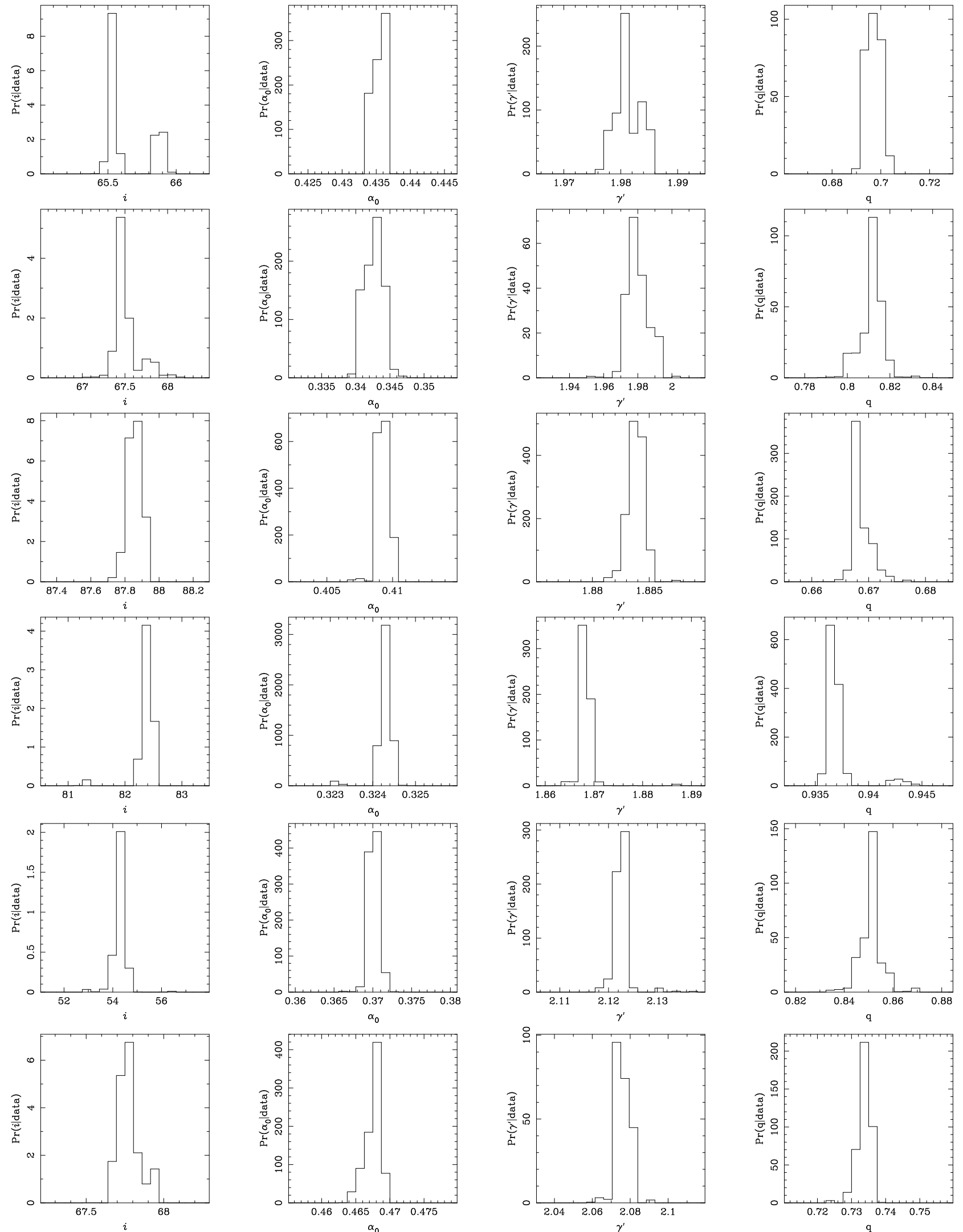

Figure 12. Marginalized posterior probability distributions of the power-law model parameters $i$ (inclination), $\gamma^{\prime}$ (logarithmic slope), $\alpha_{0}$ (lens strength) and $q$ (axial ratio) for each of the analyzed systems, obtained from the nested sampling evidence exploration (see text). From top to bottom, the uncertainties correspond to galaxies SDSS J0037, SDSS J0216, SDSS J0912, SDSS J0959, SDSS J1627 and SDSS J2321. 


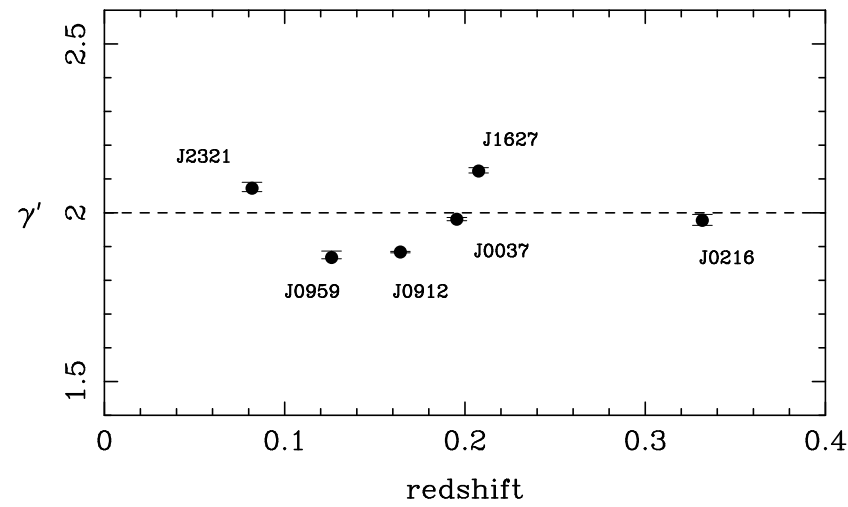

Figure 13. The logarithmic slope of the total density profile plotted against redshift for the six early-type lens galaxies in the ensemble. The dashed line indicates the slope $\gamma^{\prime}=2$, corresponding to the isothermal profile. The error bars are calculated by considering the region of the marginalized posterior PDF for $\gamma^{\prime}$ (see Fig. 12] which contains 99 per cent of the probability.

Inserting the values for $\gamma_{\mathrm{i}}^{\prime}$ and $\delta \gamma_{\mathrm{i}}^{\prime}$ determined in our analysis, we solve Eq. [8] to find, for our sample of six distant ellipticals, an intrinsic spread $\sigma_{\gamma^{\prime}}=0.092_{-0.005}^{+0.089}$ around the average logarithmic slope, corresponding to $4.6_{-0.2}^{+4.5}$ per cent. A measure of the joint posterior of these quantities is provided by Fig. 14, where we plot $\mathcal{P}\left(\gamma_{\mathrm{c}}^{\prime}, \sigma_{\gamma^{\prime}}\right)$ and we draw contours corresponding to posterior (or likelihood in the case of flat prior) ratios $\mathcal{P} / \mathcal{P}_{\max }=e^{-\Delta \tilde{\chi}^{2} / 2}$, with $\Delta \tilde{\chi}^{2}=1,4,9$. We note that these contours are only for indication, and formally have a proper meaning only in the case of a multivariate Gaussian, in which case $\tilde{\chi}^{2}$ is the usual chi-square.

If we consider a different prior in Eq. (7), the outcome is only slightly modified. For instance, if we adopt $p \propto 1 / \sigma_{\gamma^{\prime}}$, which formalizes the absence of a priori information on the scale (i.e. the order of magnitude) of $\sigma_{\gamma^{\prime}}$, we find a maximum likelihood value of 0.085 . This points out that, despite the fact that we only have a handful of systems, the results are essentially driven by the data, with the choice of the prior playing only a minimal role.

\subsection{Axial ratio of the density distribution}

The axial ratio $q$ of the total density distribution is found to be always rounder than $\sim 0.65$. It is interesting to compare this quantity with the intrinsic axial ratio $q_{\star}$ of the luminous distribution, obtained by deprojecting the observed isophotal axial ratio $q_{\star, 2 \mathrm{D}}$, i.e.

$q_{\star}=\sqrt{1-\left(1-q_{\star, 2 \mathrm{D}}^{2}\right) / \sin ^{2} i}$,

where we use the best model value for the inclination $i$. The results are illustrated in Fig. 15. For four of the galaxies in the sample, the flattenings coincide closely, while the total distribution is rounder in the case of SDSS J1627 and SDSS J0959. The discrepancy is particularly conspicuous for SDSS J0959, where $q / q_{\star}=1.4$, while it is only $\sim 1.1$ for SDSS J1627. Intriguingly, SDSS J0959 is the only clearly fast-rotating galaxy in the sample (see the velocity map in Fig. 8 and the strongly asymmetric DF in Fig. 16(d), and has also peculiar dynamical properties when compared with the rest of the ensemble, as discussed in Section 5

\section{RECOVERED DYNAMICAL STRUCTURE}

As reviewed in Section 3 for any given total gravitational potential the CAULDRON algorithm determines the best-fitting dynamical

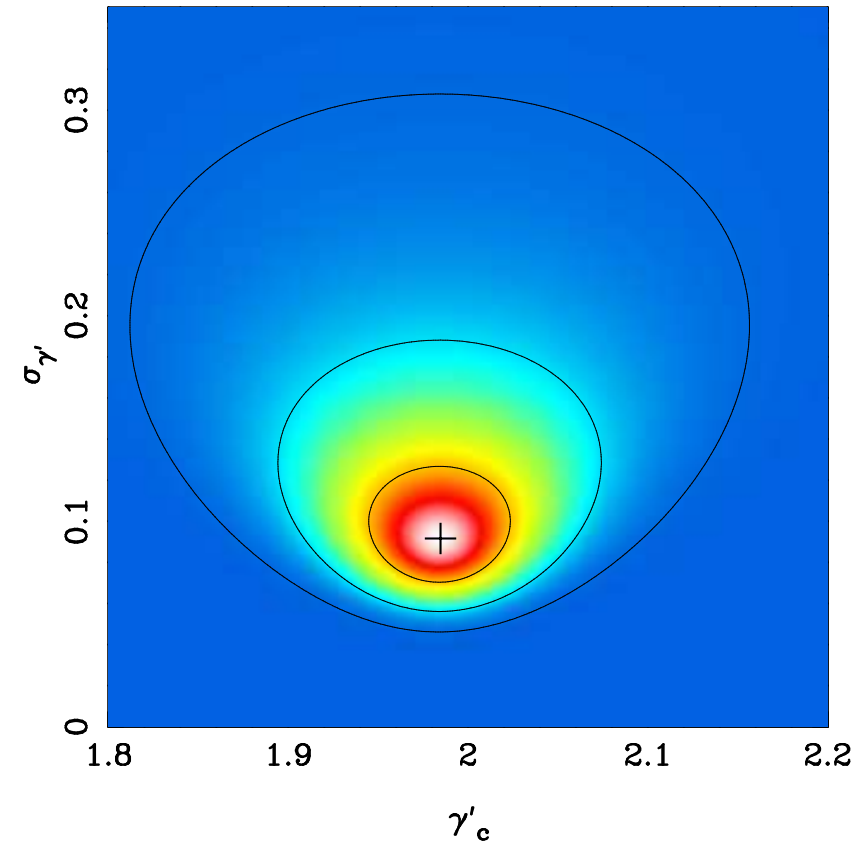

Figure 14. The map shows the joint posterior probability, given by Eq. 7), as a function of $\gamma_{\mathrm{c}}^{\prime}$ and $\sigma_{\gamma^{\prime}}$. The cross marks the position of the maximum. The contours correspond to posterior ratios $\mathcal{P} / \mathcal{P}_{\max }=e^{-\Delta \tilde{\chi}^{2} / 2}$, with $\Delta \tilde{\chi}^{2}=$ $1,4,9$ (see text).

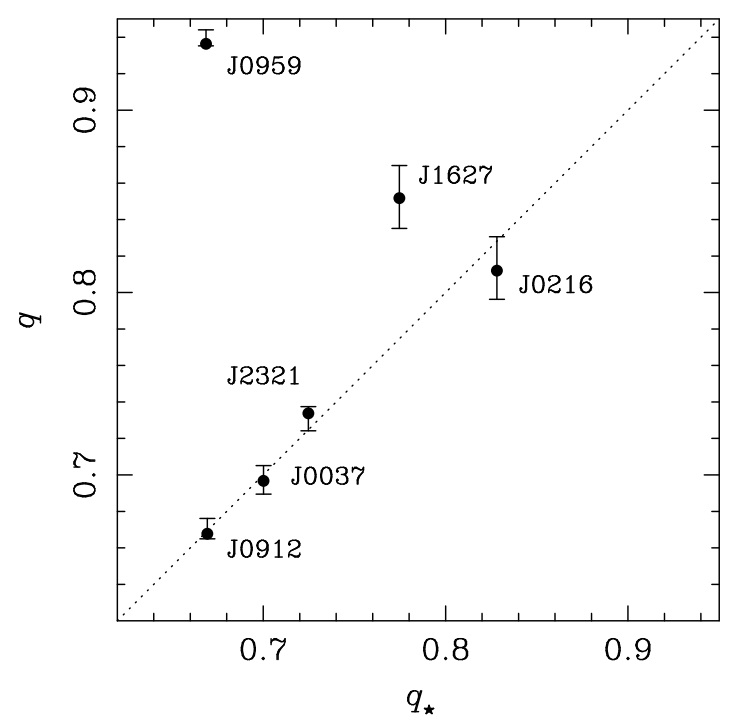

Figure 15. Axial ratio $q$ of the total density distribution plotted against the intrinsic axial ratio $q_{\star}$ of the luminous distribution. For each galaxy, $q_{\star}$ has been calculated from the corresponding observed isophotal axis ratio $q_{\star, 2 \mathrm{D}}$, by adopting the best-model recovered inclination. The error bars are calculated by considering the region of the marginalized posterior PDF for $q$ (see Fig. 12] which contains 99 per cent of the probability.

model by means of TICs superposition. Therefore, the best model of each galaxy has an associated best reconstructed map of the relative TICs weights, which is a representation in the integral space $\left(E, L_{z}\right)$ of the corresponding (weighted) two-integral DF. The maps are shown in Fig. 16 In this work, consistently with C08, we employ a library of 100 TICs for the dynamical modelling. The TIC grid is constructed by considering $N_{E}=10$ elements logarithmically sampled in the circular radius $R_{\mathrm{c}}$, and, for each of them, 


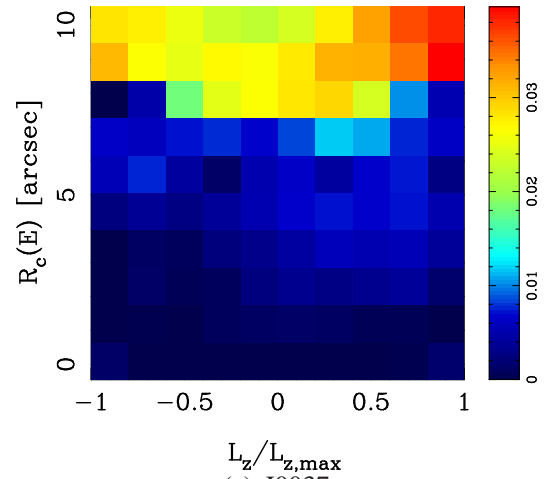

(a) J0037

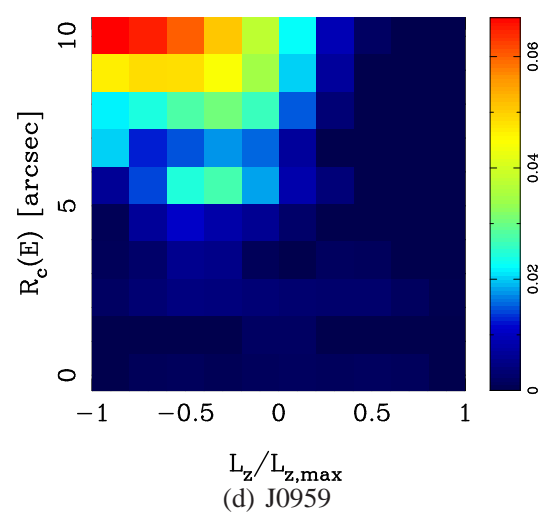

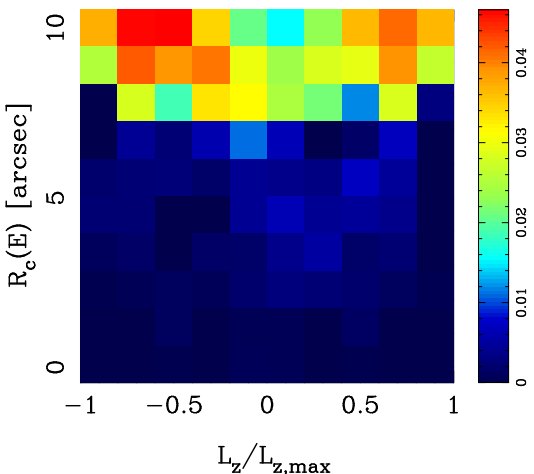

(b) J0216

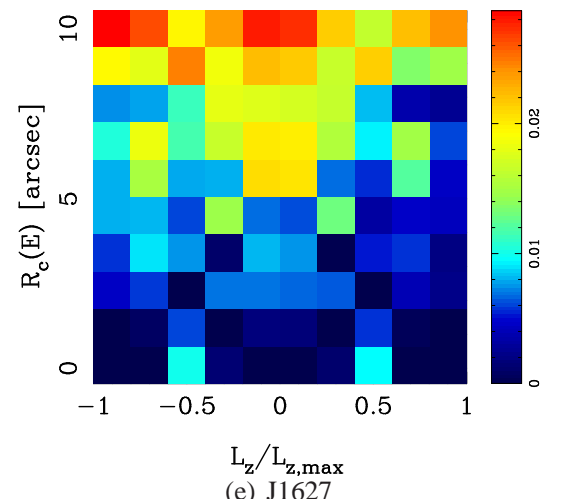

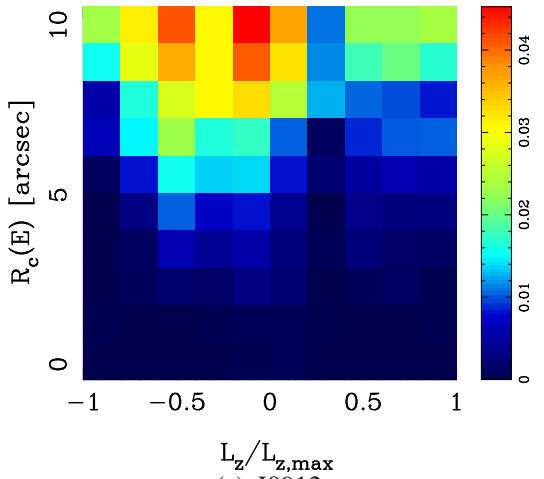

(c) J0912

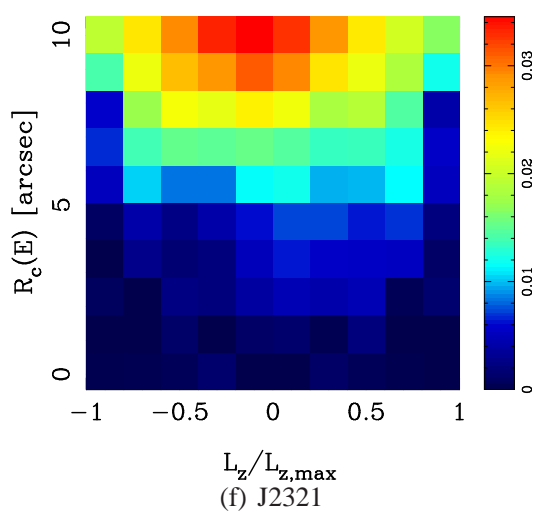

Figure 16. Best model reconstruction of the weighted two-integral DFs of the sample galaxies. The value of each pixel represents the relative contribution of the corresponding TIC to the total mass of the galaxy.

$N_{L_{z}}=5$ elements linearly sampled in angular momentum between $L_{z}=0$ and $L_{z}=L_{z, \max }\left(R_{\mathrm{c}}\right)$, mirrored in the negative $L_{z}$ value 6 . Even though this grid may appear coarse due to the limited number of TICs employed, it represents - for the applications described in this paper-an excellent compromise between computational efficiency and quality of the observables reconstruction. Increasing the number of grid elements has the effect of improving the surface brightness reconstruction, but does not change significantly the values of the recovered parameters. It also makes the reconstructed TIC weights map smoother, while preserving the main features already visible in the corresponding coarse map.

While the phase-space DF completely determines the structure and dynamics of a (collisionless) stellar system, it is not immediately intuitive to interpret. Therefore, from the best reconstructed DFs we derive dynamical characterizations of the galaxies, such as the anisotropy parameters $(\$ 5.1)$ the $(v / \sigma, \epsilon)$ diagram $(\S[5.2)$, and

${ }^{6}$ The grid in the radial coordinate $R_{\mathrm{c}}$ corresponds to a grid in the energy $E_{\mathrm{c}}$, where

$E_{\mathrm{c}} \equiv E\left(R_{\mathrm{c}}\right)=\Phi\left(R_{\mathrm{c}}, 0\right)+\frac{v_{\mathrm{c}}^{2}\left(R_{\mathrm{c}}\right)}{2}$,

and the circular speed is given by:

$v_{\mathrm{c}}^{2}\left(R_{\mathrm{c}}\right)=\left.R_{\mathrm{c}} \frac{\partial \Phi}{\partial R}\right|_{\left(R_{\mathrm{c}}, 0\right)}$

The choice for the circular radius also sets the corresponding maximum angular momentum along the $z$ axis: $L_{z, \text { max }}\left(R_{\mathrm{c}}\right)=R_{\mathrm{c}} v_{\mathrm{c}}\left(R_{\mathrm{c}}\right)$. the angular momentum $(\S 5.3$ which can be more easily related with the observations.

\subsection{Anisotropy parameters}

The global anisotropy of an elliptical galaxy is related to the distribution of its stellar orbits and is often considered an important indicator of the assembly mechanism of the system (see e.g. Burkert \& Naab 2005).

For an axisymmetric system, the global shape of the velocity dispersion tensor can be quantified by using the three anisotropy parameters (Cappellari et al. 2007; Binney \& Tremaine 2008)

$\beta \equiv 1-\frac{\Pi_{z z}}{\Pi_{R R}}$

$\gamma \equiv 1-\frac{\Pi_{\varphi \varphi}}{\Pi_{R R}}$,

and

$\delta \equiv 1-\frac{2 \Pi_{z z}}{\Pi_{R R}+\Pi_{\varphi \varphi}}=\frac{2 \beta-\gamma}{2-\gamma}$,

where

$\Pi_{k k}=\int \rho \sigma_{k}^{2} \mathrm{~d}^{3} x$

denotes the total unordered kinetic energy in the coordinate direction $k$, and $\sigma_{k}$ is the velocity dispersion along the direction $k$ at any given location in the galaxy. For an isotropic system (e.g. the classic isotropic rotator) the three parameters are all zero. Stellar systems supported by a two-integral DF, as assumed in our case, are 
isotropic in the meridional plane, i.e. $\sigma_{R}^{2}=\sigma_{z}^{2}$ everywhere, which implies $\beta \equiv 0$.

For each object in the sample, we have calculated the anisotropy parameters by integrating up to half the effective radius, which is the typical region inside which the galaxy models are more strongly constrained. The results are reported in Table 4 All the systems, with the exception of SDSS J0959, have slightly positive $\delta$, i.e. are mildly anisotropic in the sense of having larger pressure parallel to the equatorial plane than perpendicular to it. This is quite similar to what Cappellari et al. (2007) and Thomas et al. (2008), using three-integral axisymmetric orbit-superposition codes, find for local ellipticals; however, their samples also display a few galaxies with clearly higher anisotropy $(\delta \sim 0.4)$. The fast-rotating galaxy SDSS J0959, instead, is anisotropic in the opposite sense, due to the fact that for this system $\sigma_{\varphi}^{2}<\sigma_{R}^{2}=\sigma_{z}^{2}$ over most of the density-weighted volume, which translates into a negative $\delta$ parameter. This property is uncommon, although not unprecedented, for nearby early-type galaxies: two systems out of the 19 analyzed by Thomas et al. (2008) have $\delta<0$, while no case is reported from the SAURON sample.

Since for our models $\beta=0$ by construction, the two remaining anisotropy parameters are univocally related by Eq. 14 so that, for $0<\delta<1, \gamma$ is necessarily negative. The two-integral DF assumption in general does not hold for nearby ellipticals (e.g. Merrifield 1991; Gerssen et al. 1997; Thomas et al. 2008); if this is the case also for distant ellipticals, then the recovered $\gamma$ could be significantly in error. On the other hand, as shown in Barnabè et al. (2008), the global parameter $\delta$ is more robust, and can be reliably recovered by CAULDRON (typically within $\sim 15$ per cent) even when the assumptions of two-integral DF and axial symmetry are both violated.

\subsection{The global and local $v / \sigma$}

The $(v / \sigma, \epsilon)$ diagram provides a classic indicator to estimate the importance of rotation with respect to random motions in earlytype galaxies (see Binney 1978). For each system, we calculate the "intrinsic" (i.e. inclination corrected) global quantity $v / \sigma$ from the best reconstructed DF by integrating up to $R_{\mathrm{e}} / 2$ (the results are listed in Table 4), and we plot it against the intrinsic ellipticity of the luminous distribution $\epsilon_{\star}=1-q_{\star}$. The diagram is presented in Fig. 17 and compared with the findings for 24 SAURON galaxies, corrected for inclination (Cappellari et al. 2007). There is a sharp dichotomy in the SLACS subsample between the obviously fastrotating system SDSS J0959 and the remaining galaxies, four of which have $v / \sigma \approx 0.2$ (two of these systems have clear characteristics of slow rotators, as discussed in Section 5.3.

Whereas $v / \sigma$ is a global quantity which provides information on the general properties of the galaxy, important insights on the characteristics of the system at different locations of the meridional plane are offered by its local analogue, i.e. the ratio $\left\langle v_{\varphi}\right\rangle / \bar{\sigma}$ between the mean rotation velocity around the $z$-axis and the mean velocity dispersion $\bar{\sigma}^{2}=\left(\sigma_{R}^{2}+\sigma_{\varphi}^{2}+\sigma_{z}^{2}\right) / 3$. We illustrate this quantity in Fig. 18 for the galaxies in our sample. For visualization purposes, the plot was produced from a weighted DF map of 900 elements ( $N_{E}=30$ and $N_{L_{z}}=15$, mirrored). In order to do this, we reoptimized the best model for the dynamics hyperparameters only, determining the new optimal level of the regularization, while all the other parameters were kept fixed. This procedure amounts to some extent to an interpolation over the weighted DF map of Fig.16 with the aim of obtaining a smoother distribution.

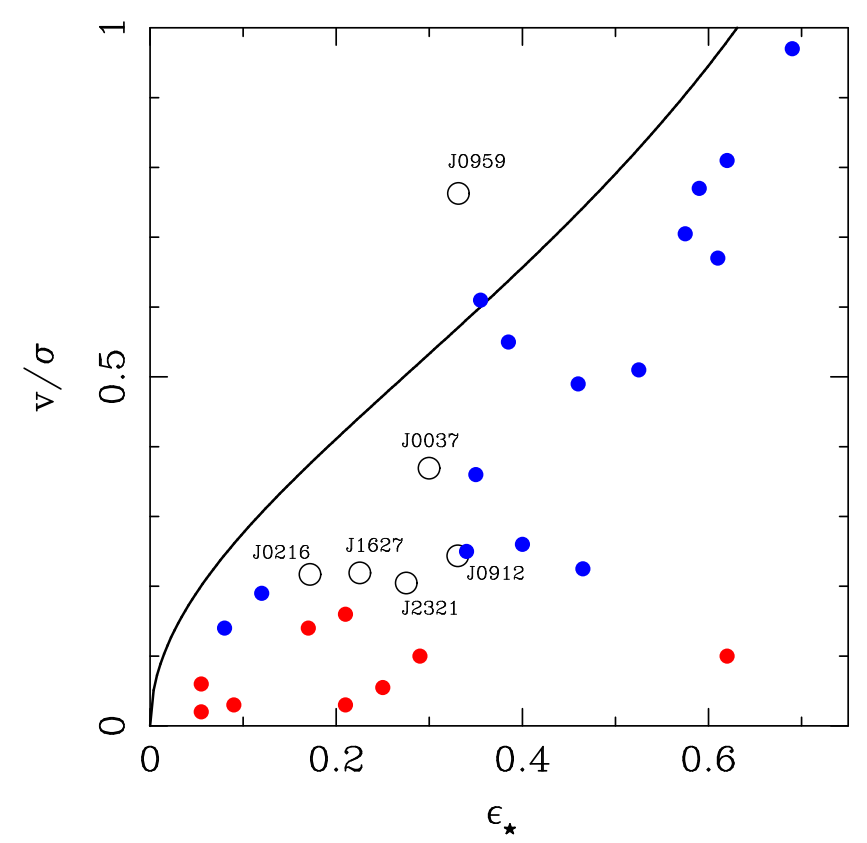

Figure 17. Model $(v / \sigma, \epsilon)$ diagram for the six lens galaxies in our sample (black circles): $\epsilon_{\star}$ is the intrinsic ellipticity of luminous distribution, and $v / \sigma$ is calculated from the best model by integrating up to $R_{\mathrm{e}} / 2$. We also show, for comparison, the corresponding quantities (corrected for inclination; $v / \sigma$ measured within $R_{\mathrm{e}}$ ) for the 24 nearby SAURON ellipticals studied in Cappellari et al. (2007), divided in fast and slow rotators (blue and red points, respectively). The solid line shows the location of edge-on isotropic rotators, assuming $\alpha=0.15$ (see Binney 2005).

\subsection{Angular momentum}

Another robust way to characterize the global velocity structure of a galaxy is provided by its angular momentum content. For each galaxy in the ensemble we calculate the (mass-normalized) component of the angular momentum parallel to the axis of symmetry as

$J_{z}=\frac{\int \rho_{\star} R\left\langle v_{\varphi}\right\rangle \mathrm{d}^{3} x}{\int \rho_{\star} \mathrm{d}^{3} x}$,

where $R$ is the radial coordinate, $\left\langle v_{\varphi}\right\rangle$ denotes the mean azimuthal stellar velocity at position $\boldsymbol{x}$ and $\rho_{\star}$ is the spatial density of stars as obtained by the best reconstructed DF, i.e. $\rho_{\star}=\int f \mathrm{~d}^{3} v$. The results obtained by integrating inside $R_{\mathrm{e}} / 2$ (the region most strongly constrained by the observations for all the systems) are reported in Table 4 in units of $\mathrm{kpc} \mathrm{km} \mathrm{s}^{-1}$.

Whereas the dimensional parameter $J_{z}$ has a direct physical interpretation as angular momentum, it is not the most practical way to quantify and to compare the level of ordered rotation in elliptical galaxies. To this purpose, we define a more convenient adimensional parameter as:

$j_{z} \equiv \frac{\int \rho_{\star} R\left|\left\langle v_{\varphi}\right\rangle\right| \mathrm{d}^{3} x}{\int \rho_{\star} R \sqrt{\left\langle v_{\varphi}\right\rangle^{2}+\bar{\sigma}^{2}} \mathrm{~d}^{3} x}$.

This quantity is effectively the intrinsic equivalent of the observationally-defined $\lambda_{R}$ parameter introduced by Emsellem et al. (2007) as an objective criterion for the kinematic classification of early-type galaxies. Analogously to $\lambda_{R}$, $j_{z}$ tends to unity for systems which display large-scale ordered rotation, and conversely it goes to zero for galaxies globally 
Table 4. Recovered dynamical quantities for the six analyzed SLACS lens galaxies.

\begin{tabular}{cccccc}
\hline Galaxy & $\delta$ & $\gamma$ & $v / \sigma$ & $J_{z}$ & $j_{z}$ \\
\hline $\mathrm{J} 0037$ & 0.16 & -0.37 & 0.37 & 112 & 0.248 \\
$\mathrm{~J} 0216$ & 0.08 & -0.17 & 0.22 & 11 & 0.116 \\
$\mathrm{~J} 0912$ & 0.07 & -0.15 & 0.24 & -231 & 0.229 \\
$\mathrm{~J} 0959$ & -0.16 & 0.27 & 0.76 & -158 & 0.645 \\
$\mathrm{~J} 1627$ & 0.16 & -0.38 & 0.23 & -69 & 0.181 \\
$\mathrm{~J} 2321$ & 0.14 & -0.32 & 0.20 & -5 & 0.075
\end{tabular}

Notes: For each galaxy we list: global anisotropy parameters $\delta$ and $\gamma(\beta=0$ by construction under the model assumption of two-integral DF); global $v / \sigma$ ratio; angular momentum along the rotation axis $J_{z}$ (in units of $\mathrm{kpc} \mathrm{km}$ $\mathrm{s}^{-1}$ ); dimensionless rotation parameter $j_{z}$. The dynamical quantities have been calculated, for each system, within a cylindrical region of radius and height equal to $R_{\mathrm{e}} / 2$.

dominated by random motions, whereas the same galaxies might have a moderate $v / \sigma$ ratio due to the presence of small-scale rotation patterns in the high-density central regions.

We have computed $j_{z}$ for the SLACS subsample by integrating inside half the effective radius, listing the results in Table 4 The small number of galaxies in the sample and the limited spatial coverage and quality of the kinematic data sets do not allow us to trace a sharp demarcation line between slow and fast rotators; nevertheless, there is a clear indication that SDSS J0959 belongs to the latter, while SDSS J0216 and SDSS J2321 are part of the first group, although they could not be straightforwardly identified as slow rotators on the basis of the $(v / \sigma, \epsilon)$ diagram only. The remaining three galaxies lie somewhat in between.

\section{SUMMARY AND CONCLUSIONS}

We have conducted, for the first time, an in-depth analysis of the mass distribution and dynamical structure of a sample of massive early-type galaxies beyond the very local Universe, with a redshift range of $z=0.08-0.33$.

The examined systems are six early-type lens galaxies from the SLACS survey for which both HST/ACS high-resolution imaging and VLT VIMOS integral field spectroscopy are available. These unique, high-quality data sets of early-type galaxies beyond the local Universe have enabled us to carry out a joint analysis of these systems, by combining gravitational lensing and stellar dynamics in a fully self-consistent way (using the specifically designed code caUlDron). The method is completely embedded within the framework of Bayesian statistics, permitting an objective data-driven determination of the "best model", given the observations and our priors (formalized by the choice of the regularization). This technique makes it possible-under the assumptions of axial symmetry and two-integral stellar DF - to disentangle to a large extent several classical degeneracies and to effectively "dissect" the investigated galaxies, recovering their intrinsic structure. We summarize and discuss as follows the main results of this study:

(i) The global density distribution of massive early-type galaxies within approximately $1 R_{\mathrm{e}}$ is remarkably well described by a simple axisymmetric power-law profile. Despite being very sensitive to the features of the underlying mass distribution (as shown by simulations of non axially symmetric systems, e.g. Barnabè et al. 2008; see also Koopmans 2005 and Vegetti \& Koopmans 2008), the lensed images can be reconstructed almost to the noise level by adopting a $\rho \propto m^{-\gamma^{\prime}}$ model (not even a weak external shear is required), indicating a surprising degree of smoothness in the mass structure of ellipticals. While this conclusion could already be envisioned from the results of the Koopmans et al. (2006) study of SLACS lenses, here we have shown that such smooth models are also consistent with the observed surface brightness and kinematics maps. We suggest that this significantly smooth structure might be related to the formation mechanisms of early-type galaxies.

(ii) The average logarithmic slope of the total mass density distribution is $\left\langle\gamma^{\prime}\right\rangle=1.98 \pm 0.05$, with an intrinsic spread of $4.6_{-0.2}^{+4.5}$ per cent. The galaxies in the sample have therefore a density profile consistent with isothermal, corresponding to flat rotation curves, inside a range of Einstein radii of $0.3-0.6 R_{\mathrm{e}}$. This is in agreement with the findings of previous studies of ellipticals both in the local Universe and up to redshift of 1 (e.g. Gerhard et al. 2001, Koopmans et al. 2006, Thomas et al. 2007).

(iii) There is no evidence for evolution of the logarithmic total density slope within the probed range of redshifts, although this is not surprising given the findings of the (non self-consistent) combined lensing and dynamics study of Koopmans et al. (2006). However, it does show that we have systematics well under control.

(iv) The shape of the total density distribution is fairly round, with an axial ratio $q \gtrsim 0.65$, and does not differ much from the intrinsic axial ratio of the luminous distribution (obtained via deprojection of the observed isophotal ratio, by making use of the recovered best model value for the inclination). The only exception is represented by the case of the lenticular galaxy SDSS J0959, which has a total density profile much rounder that the luminous one.

(v) The lower limit for the dark matter fraction, calculated within spherical shells under the hypotheses of "maximum bulge" and position-independent stellar mass-to-light ratio, falls in the range $10-25$ per cent (of the total mass) at half the effective radius, and rises to $15-30$ per cent at $r=R_{\mathrm{e}}$. This is fully consistent with the results of several studies of the dark and luminous mass distribution in nearby ellipticals (in particular Gerhard et al. 2001; Cappellari et al. 2006; Thomas et al. 2007).

(vi) The SLACS galaxies in our subsample are only mildly anisotropic, with the global parameter $-0.16 \leqslant \delta \leqslant 0.16$. Five out of six systems are slightly flattened (beyond the contribution of rotation) by having a larger pressure support parallel to the equatorial plane rather than perpendicular to it; the situation is reversed in the case of SDSS J0959, which shows negative $\delta$.

(vii) From the inspection of the stellar velocity maps, the global and local $v / \sigma$ ratio and, more decisively, the intrinsic rotation parameter $j_{z}$ (directly related to the angular momentum of the stellar component of the galaxy), it is possible to objectively quantify the level of ordered rotation with respect to the random motions. Two of the systems, namely SDSS J0216 and SDSS J2321, are identified as slow rotators, while SDSS J0959 unambiguously shows the characteristics of fast rotators, including the relatively low mass and luminosity: with $M_{B}=-20.58$ it is the only system in our sample to lie within the typical range of absolute magnitudes for fast rotators determined by Emsellem et al. (2007). The remaining three galaxies exhibit a moderate amount of rotation.

Overall, the early-type lens galaxies analyzed in this paper, in the redshift range $z=0.08-0.33$, are found to be very similar to the local massive ellipticals, in terms of structural and dynamical properties of their inner regions. Our study shows, for the first time, that also the physical distinction between slow and fast rotators 


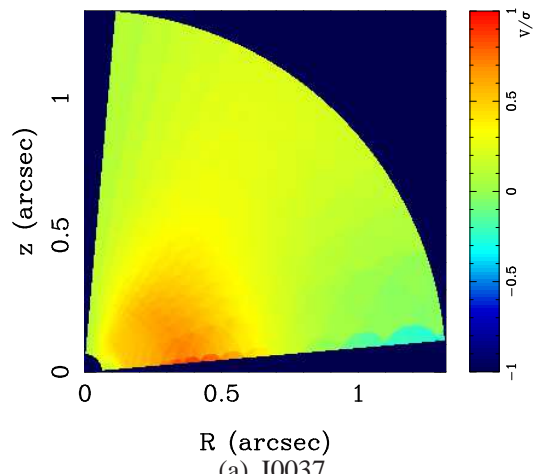

(a) $\mathrm{J} 0037$

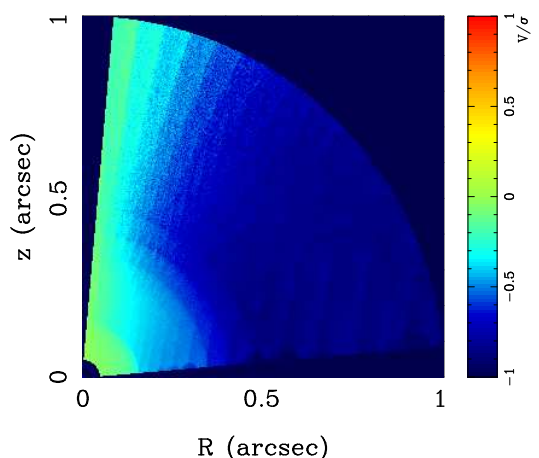

(d) J0959

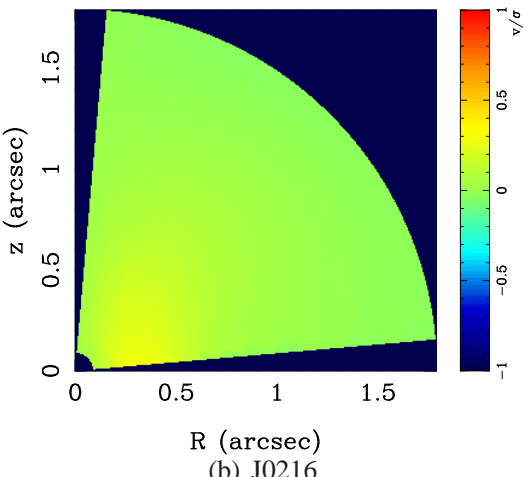

(b) J0216

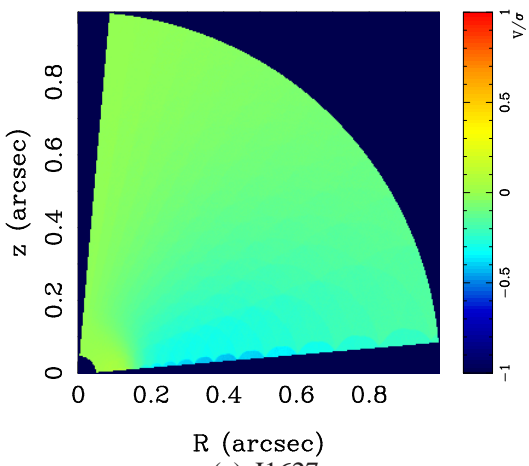

(e) J1627

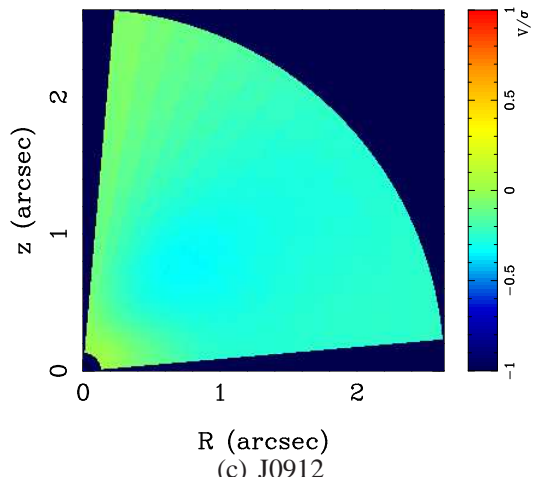

(c) J0912

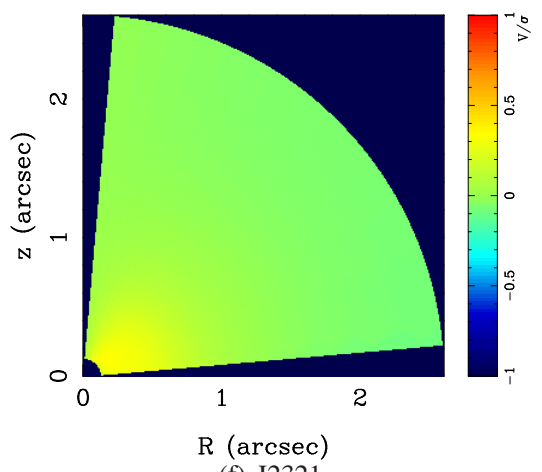

(f) $\mathrm{J} 2321$

Figure 18. Maps of the local $\left\langle v_{\varphi}\right\rangle / \bar{\sigma}$ ratio between the mean rotation velocity around the $z$-axis and the mean velocity dispersion, plotted up to $R_{\mathrm{e}} / 2$ in the positive quadrant of the meridional plane.

(originally revealed by the SAURON survey: Emsellem et al.2007; Cappellari et al.2007) is already in place at redshift $\gtrsim 0.1$, although a larger sample is necessary in order to quantify this more precisely to higher redshifts. Since a series of studies (Treu et al. 2006; Bolton et al. 2008; Treu et al. 2008) has shown that the SLACS systems are statistically identical—in terms of observational properties and environment - to non-lens galaxies of comparable size and luminosity, we can generalize the results of this work and conclude that at least the most massive elliptical galaxies did not experience any major evolutionary process in their global structural properties between redshift 0 and 0.3 . Since the look-back time is only 3.7 Gyr, this might not be surprising. However, pushing lensing and dynamics techniques back in redshift and cosmic time is crucial if we ever wish to fully understand the structural evolution of earlytype galaxies. In this paper a first step has been taken, using more sophisticated self-consistent techniques. This goes beyond what is possible with the use of lensing or dynamics alone.

On the other hand, we also find differences between the analyzed systems and nearby galaxies when we compare the respective global anisotropy parameters. Although the values of $\delta$ recovered for the SLACS subsample fall within the typical range for local galaxies, we note that we do not find any systems with $\delta \gtrsim 0.20$, which are instead quite common in the SAURON and Coma samples. This might be due, however, to the very modest size of our sample, particularly in terms of fast-rotating objects. A much more drastic discrepancy is evident in the distributions of the anisotropy parameters $\gamma$ and $\beta$, plausibly due to the limitations of our assumption of two-integral DF, which imposes $\sigma_{R}^{2}=\sigma_{z}^{2}$ at every location. Isotropy in the meridional plane is not observed, in general, for local ellipticals, where usually $\sigma_{R}^{2}>\sigma_{z}^{2}$ (e.g. Gerssen et al.
1997; Thomas et al. 2008). If this applies also to more distant systems, then the anisotropy will not be correctly estimated by our method, and more sophisticated axisymmetric three-integral models might provide a better dynamical description of the galaxy. We foresee future developments of the CAULDRON algorithm in this direction, concurrently with the availability of improved kinematic data sets. As for the present analysis, however, the $f\left(E, L_{z}\right)$ assumption generally appears to work satisfactorily, providing generally correct reconstructions of the observables. Furthermore, as tested in Barnabè et al. (2008), the current method is robust enough to recover in a reliable way several important global properties of the analyzed galaxy (such as the density slope, dark matter fraction, angular momentum and $\delta$ parameter, although not the flattening and the $\gamma$ and $\beta$ parameters) even if it is applied to a complex, non-symmetric system which departs significantly from the idealized assumptions of axisymmetry and two- or three-integral DF, and which is likely more extreme than the typical ellipticals under study.

In future papers in this series, we plan to extend the current study by applying the combined analysis to all the 30 systems for which two-dimensional kinematic maps are or will become available. This includes the entire sample of 17 SLACS early-type lens galaxies for which integral field spectroscopy has been obtained. Two-dimensional kinematic maps can also be obtained for a further 13 lens galaxies for which long-slit spectroscopic observations have been conducted with the instrument LRIS mounted on Keck-I, with the slit positions aligned with the major axis of the system and offset along the minor axis in order to mimic integral field spectroscopy. 


\section{ACKNOWLEDGMENTS}

M. B. is grateful to Mattia Righi for many lively and stimulating discussions. M. B. acknowledges the support from an NWO program subsidy (project number 614.000.417). OC and LVEK are supported (in part) through an NWO-VIDI program subsidy (project number 639.042.505). We also acknowledge the continuing support by the European Community's Sixth Framework Marie Curie Research Training Network Programme, Contract No. MRTN-CT-2004-505183 'ANGLES'. TT acknowledges support from the NSF through CAREER award NSF-0642621, by the Sloan Foundation through a Sloan Research Fellowship, and by the Packard Foundation through a Packard Fellowship. Support for programs \#10174 and \#10494 was provided by NASA through a grant from the Space Telescope Science Institute, which is operated by the Association of Universities for Research in Astronomy, Inc., under NASA contract NAS 5-26555.

\section{REFERENCES}

Arnaboldi M., Freeman K. C., Mendez R. H., Cappaccioli M., Ciardullo R., Ford H., Gerhard O., Hui X., Jacoby G. H., Kudritzki R. P., Quinn P. J., 1996, ApJ, 472, 145

Barnabè M., Koopmans L. V. E., 2007, ApJ, 666, 726

Barnabè M., Nipoti C., Koopmans L. V. E., Vegetti S., Ciotti L., 2008, MNRAS, in press

Barnes J. E., 1992, ApJ, 393, 484

Barth A. J., Ho L. C., Sargent W. L. W., 2002, AJ, 124, 2607

Bertin E., 2008, SWARP User's Guide. v2.17.0 edn

Bertin G., Bertola F., Buson L. M., Danzinger I. J., Dejonghe H., Sadler E. M., Saglia R. P., de Zeeuw P. T., Zeilinger W. W., 1994, A\&A, 292, 381

Binney J., 1978, MNRAS, 183, 501

Binney J., 2005, MNRAS, 363, 937

Binney J., Tremaine S., 2008, Galactic Dynamics. Princeton University Press

Bolton A. S., Burles S., Koopmans L. V. E., Treu T., Gavazzi R., Moustakas L. A., Wayth R., Schlegel D. J., 2008, ApJ, 682, 964

Bolton A. S., Burles S., Koopmans L. V. E., Treu T., Moustakas L. A., 2006, ApJ, 638, 703

Bolton A. S., Treu T., Koopmans L. V. E., Gavazzi R., Moustakas L. A., Burles S., Schlegel D. J., Wayth R., 2008, ApJ, 684, 248

Borriello A., Salucci P., Danese L., 2003, MNRAS, 341, 1109

Burkert A., Naab T., 2005, MNRAS, 363, 597

Cappellari M., Bacon R., Bureau M., Damen M. C., Davies R. L., de Zeeuw P. T., Emsellem E., Falcón-Barroso J., et al., 2006, MNRAS, 366, 1126

Cappellari M., Emsellem E., 2004, PASP, 116, 138

Cappellari M., Emsellem E., Bacon R., Bureau M., Davies R. L., de Zeeuw P. T., Falcón-Barroso J., Krajnović D., Kuntschner H., McDermid R. M., Peletier R. F., Sarzi M., van den Bosch R. C. E., van de Ven G., 2007, MNRAS, 379, 418

Carollo C. M., de Zeeuw P. T., van der Marel R. P., Danziger I. J., Qian E. E., 1995, ApJ, 441, L25

Chandrasekhar S., 1969, Ellipsoidal Figures of Equilibrium. Yale University Press

Cole S., Lacey C. G., Baugh C. M., Frenk C. S., 2000, MNRAS, 319, 168

Côté P., McLaughlin D. E., Cohen J. G., Blakeslee J. P., 2003, ApJ, 591, 850

Cousins R. D., 1995, American Journal of Physics, 63, 398
Cretton N., de Zeeuw P. T., van der Marel R. P., Rix H.-W., 1999, ApJS, 124, 383

Czoske O., Barnabè M., Koopmans L. V. E., 2008, in Probing Stellar Populations out to the Distant Universe Conference, (arXiv:0811.2391) Integral-field spectroscopy of SLACS lenses

Czoske O., Barnabè M., Koopmans L. V. E., Treu T., Bolton A. S., 2008, MNRAS, 384, 987

de Lorenzi F., Gerhard O., Saglia R. P., Sambhus N., Debattista V. P., Pannella M., Méndez R. H., 2008, MNRAS, 385, 1729

de Zeeuw P. T., Bureau M., Emsellem E., Bacon R., Carollo C. M., Copin Y., Davies R. L., Kuntschner H., et al., 2002, MNRAS, 329, 513

Emsellem E., Cappellari M., Krajnović D., van de Ven G., Bacon R., Bureau M., Davies R. L., de Zeeuw P. T., et al., 2007, MNRAS, 379, 401

Emsellem E., Cappellari M., Peletier R. F., McDermid R. M., Bacon R., Bureau M., Copin Y., Davies R. L., Krajnović D., Kuntschner H., Miller B. W., de Zeeuw P. T., 2004, MNRAS, 352,721

Fabbiano G., 1989, ARAA, 27, 87

Falco E. E., Gorenstein M. V., Shapiro I. I., 1985, ApJ, 289, L1

Franx M., van Gorkom J. H., de Zeeuw T., 1994, ApJ, 436, 642

Fukazawa Y., Botoya-Nonesa J. G., Pu J., Ohto A., Kawano N., 2006, ApJ, 636, 698

Gavazzi R., Treu T., Koopmans L. V. E., Bolton A. S., Moustakas L. A., Burles S., Marshall P. J., 2008, ApJ, 677, 1046

Gavazzi R., Treu T., Rhodes J. D., Koopmans L. V. E., Bolton A. S., Burles S., Massey R. J., Moustakas L. A., 2007, ApJ, 667, 176

Gerhard O., Kronawitter A., Saglia R. P., Bender R., 2001, AJ, 121, 1936

Gerhard O. E., 1993, MNRAS, 265, 213

Gerssen J., Kuijken K., Merrifield M. R., 1997, MNRAS, 288, 618

Humphrey P. J., Buote D. A., Gastaldello F., Zappacosta L., Bullock J. S., Brighenti F., Mathews W. G., 2006, ApJ, 646, 899

Jesseit R., Naab T., Peletier R. F., Burkert A., 2007, MNRAS, 376, 997

Kelson D. D., Illingworth G. D., van Dokkum P. G., Franx M., 2000, ApJ, 531, 159

Kochanek C. S., 1991, ApJ, 373, 354

Koopmans L., Treu T., Bolton A. S., Burles S., Moustakas L. A., 2006, ApJ, 649, 599

Koopmans L. V. E., Treu T., 2002, ApJ, 568, L5

Koopmans L. V. E., Treu T., 2003, ApJ, 583, 606

Koopmans Léon V. E., 2005, MNRAS, 363, 1136

Kronawitter A., Saglia R. P., Gerhard O., Bender R., 2000, A\&AS, 144, 53

Loewenstein M., White III R. E., 1999, ApJ, 518, 50

MacKay D. J. C., 1992, PhD Thesis

MacKay D. J. C., 1999, Neural Comp, 11, 1035

MacKay D. J. C., 2003, Information Theory, Inference and Learning Algorithms. Cambridge University Press

Marshall P. J., Treu T., Melbourne J., Gavazzi R., Bundy K., Ammons S. M., Bolton A. S., Burles S., Larkin J. E., Le Mignant D., Koo D. C., Koopmans L. V. E., Max C. E., Moustakas L. A., Steinbring E., Wright S. A., 2007, ApJ, 671, 1196

Matsushita K., Makishima K., Ikebe Y., Rokutanda E., Yamasaki N., Ohashi T., 1998, ApJ, 499, L13

Merrifield M. R., 1991, AJ, 102, 1335

Mould J. R., Oke J. B., de Zeeuw P. T., Nemec J. M., 1990, AJ, 99, 1823 
Naab T., Khochfar S., Burkert A., 2006, ApJ, 636, L81

Rix H.-W., de Zeeuw P. T., Cretton N., van der Marel R. P., Carollo C. M., 1997, ApJ, 488, 702

Romanowsky A. J., Douglas N. G., Arnaboldi M., Kuijken K., Merrifield M. R., Napolitano N. R., Capaccioli M., Freeman K. C., 2003, Science, 301, 1696

Saglia R. P., Bertin G., Stiavelli M., 1992, ApJ, 384, 433

Schneider P., Ehlers J., Falco E. E., 1992, Gravitational Lenses. Berlin, Springer-Verlag

Schwarzschild M., 1979, ApJ, 232, 236

Scodeggio M., Franzetti P., Garilli B., Zanichelli A., Paltani S., Maccagni D., Bottini D., Le Brun V., et al., 2005, PASP, 117, 1284

Sivia D. S., Skilling J., 2006, Data Analysis: A Bayesian Tutorial. Oxford University Press

Skilling J., 2004, in Fischer R., Preuss R., Toussaint U. V., eds, American Institute of Physics Conference Series Vol. 735 of American Institute of Physics Conference Series, Nested Sampling. pp 395-405

Suyu S. H., Marshall P. J., Hobson M. P., Blandford R. D., 2006, MNRAS, 371, 983

Thomas J., Jesseit R., Naab T., Saglia R. P., Burkert A., Bender R., 2007, MNRAS, 381, 1672

Thomas J., Jesseit R., Saglia R. P., Bender R., Burkert A., Corsini E. M., Gebhardt K., Magorrian J., Naab T., Thomas D., Wegner G., 2008, MNRAS, in press

Thomas J., Saglia R. P., Bender R., Thomas D., Gebhardt K., Magorrian J., Corsini E. M., Wegner G., 2007, MNRAS, 382, 657

Toomre A., 1977, in Tinsley B. M., Larson R. B., eds, Evolution of Galaxies and Stellar Populations Mergers and Some Consequences. p. 401

Treu T., Gavazzi R., Gorecki A., Marshall P. J., Koopmans L. V. E., Bolton A. S., Moustakas L. A., Burles S., 2008, ApJ, in press

Treu T., Koopmans L. V. E., 2002, MNRAS, 337, L6

Treu T., Koopmans L. V. E., 2003, MNRAS, 343, L29

Treu T., Koopmans L. V. E., 2004, ApJ, 611, 739

Treu T., Koopmans L. V. E., Bolton A. S., Burles S., Moustakas L., 2006, ApJ, 640, 662

Trott C. M., Treu T., Koopmans L. V. E., Webster R. L., 2008, preprint (arXiv:0812.0748)

Trujillo I., Burkert A., Bell E. F., 2004, ApJ, 600, L39

Valdes F., Gupta R., Rose J. A., Singh H. P., Bell D. J., 2004, ApJS, 152, 251

van Albada T. S., Sancisi R., 1986, Royal Society of London Philosophical Transactions Series A, 320, 447

van de Ven G., Falcon-Barroso J., McDermid R. M., Cappellari M., Miller B. W., de Zeeuw P. T., 2008, preprint (arXiv:0807.4175)

van der Marel R. P., Franx M., 1993, ApJ, 407, 525

Vegetti S., Koopmans L. V. E., 2008, MNRAS, in press

Verolme E. K., de Zeeuw P. T., 2002, MNRAS, 331, 959

White S. D. M., Frenk C. S., 1991, ApJ, 379, 52

Wucknitz O., 2002, MNRAS, 332, 951

Zanichelli A., Garilli B., Scodeggio M., Franzetti P., Rizzo D., Maccagni D., Merighi R., Picat J.-P., et al., 2005, PASP, 117, 1271 